\title{
Perturbative F-theory 10-brane and M-theory 5-brane
}

\author{
Machiko Hatsuda ${ }^{a, b}$ and Warren Siegel ${ }^{c}$ \\ ${ }^{a}$ Department of Radiological Technology, Faculty of Health Science, Juntendo University, \\ Yushima, Bunkyou-ku, Tokyo 113-0034, Japan \\ ${ }^{b}$ KEK Theory Center, High Energy Accelerator Research Organization, \\ Tsukuba, Ibaraki 305-0801, Japan \\ ${ }^{c}$ C.N. Yang Institute for Theoretical Physics, State University of New York, \\ Stony Brook, NY 11794-3840, U.S.A. \\ E-mail: mhatsuda@juntendo.ac.jp, siegel@insti.physics.sunysb.edu
}

ABSTRACT: The exceptional symmetry is realized perturbatively in F-theory which is the manifest U-duality theory. The $\mathrm{SO}(5,5) \mathrm{U}$-duality symmetry acts on both the 16 spacetime coordinates and the 10 worldvolume coordinates. Closure of the Virasoro algebra requires the Gauss law constraints on the worldvolume. This set of current algebras describes a F-theory 10-brane. The $\mathrm{SO}(5,5)$ duality symmetry is enlarged to the $\mathrm{SO}(6,6)$ symmetry in the Lagrangian formulation. We propose actions of the F-theory 10-brane with $\mathrm{SO}(5,5)$ and $\mathrm{SO}(6,6)$ symmetries. The gauge fields of the latter action are coset elements of $\mathrm{SO}(6,6) / \mathrm{SO}(6 ; \mathbb{C})$ which include both the $\mathrm{SO}(5,5) / \mathrm{SO}(5 ; \mathbb{C})$ spacetime backgrounds and the worldvolume backgrounds. The $\mathrm{SO}(5,5)$ current algebra obtained from the Pasti-Sorokin-Tonin M5-brane Lagrangian leads to the theory behind M-theory, namely F-theory. We also propose an action of the perturbative M-theory 5-brane obtained by sectioning the worldvolume of the F-theory 10-brane.

KEYwords: F-Theory, M-Theory, p-branes, String Duality

ArXiv EPrint: 2107.10568 


\section{Contents}

1 Introduction 1

2 Introduction to F-theory 4

$\begin{array}{lll}3 & \mathrm{SO}(5,5) & \text { current algebra }\end{array}$

$3.1 \mathrm{SO}(5,5)$ spinor representation 6

$3.2 \mathrm{GL}(5)$ tensor representation 9

4 F-theory 10-brane actions $\quad 14$

4.1 Double zweibein formulation in T-theory 15

$\begin{array}{lll}4.2 & \mathrm{SO}(5,5) \text { Hamiltonian form action } & 17\end{array}$

$\begin{array}{lll}4.3 & \mathrm{SO}(6,6) \text { Lagrangian form action } & 18\end{array}$

4.4 GL(6) and GL(5) actions 22

5 Perturbative M-theory 5-brane action 25

6 Conclusions 28

$\begin{array}{lr}\text { A Indices } & 29\end{array}$

$\begin{array}{ll}\text { B Brackets } & 29\end{array}$

$\begin{array}{ll}\text { C 11-dimensional tensor representation } & 30\end{array}$

D Double vielbein formulation 31

\section{Introduction}

Duality is a cornucopia of string theory creating its unique properties. Superstring theory is considered to be a candidate for a unified theory of all forces, and five types of superstring theories have been shown to exist. Five superstring theories together with M-theory form pairs related by T-duality or S-duality. Then why are superstring theories related in a way of chain of dualities? T-duality is the equivalence under the interchange of $R \leftrightarrow \alpha^{\prime} / R$ for the radius of the compactified space $R$, and S-duality is the equivalence under the interchange of $g \leftrightarrow 1 / g$ for the string coupling $g$. So they relate paired theories. These T-duality and S-duality are encompassed by U-duality. Therefore we examine whether a theory with manifest U-duality exists which describes different superstring theories by its different sections. We call such a theory with manifest U-duality "F-theory". 
The theory with manifest T-duality was presented in [1-3] which was named "T-theory" later. "T-theory" was defined by the $\mathrm{O}(D, D)$ current algebra which generates gauge symmetries of background gauge fields. It contains winding modes even in an uncompactified space, and the $D$-dimensional space includes time direction to describe dynamical gravity. All string modes including massive modes are described in the T-theory which has been developed [1-11]. Section conditions eliminate winding modes while the string field theory condition $L_{0}=\bar{L}_{0}$ mixes massive winding modes with massive oscillator states [12].

Duality relates nonperturbative states of classical string theory. When duality symmetry is represented linearly in some classical theory, nonperturbative states are described perturbatively. The $\mathrm{O}(D, D)$ T-duality symmetry is represented linearly in the Double Field Theory (DFT) $[1-3,12-18]$ and the generalized geometry gives a mathematical framework of geometry induced by T-duality [19-21]. They are theories of massless modes of string theory.

T-duality and S-duality are unified into U-duality by the exceptional symmetry group which involves non-perturbative branes [22]. M-theory was conjectured as a theory to unify superstring theories through dualities whose low energy effective theory is the 11dimensional supergravity theory [23]. F-theory was firstly proposed by Vafa [24] to understand the IIB theory in the string duality web where similar ideas are also referred $[25,26]$. The $\mathrm{O}(D, D)$ T-duality symmetry is extended to the exceptional symmetry group U-duality symmetry in generalized geometry for M-theory [27, 28] and Exceptional Field Theory (EFT) [29-38]. Current algebras for branes were calculated to present generalized brackets and derive background gauge symmetries [39-41] corresponding to the U-duality covariant formulation of the 11-dimensional supergravity [29, 30].

Recently "F-theory" as a theory with manifest U-duality has been realized [7, 42-51] as a generalization of T-theory. F-theory is defined by the exceptional group current algebras on branes. The exceptional group acts both the spacetime coordinates and the worldvolume coordinates. Since the exceptional group includes both the $\mathrm{O}(D, D)$ T-duality symmetry and the $\mathrm{SL}(2 ; \mathbb{R})$ S-duality symmetry, F-theory reduces both the IIB theory and M-theory directly by sectioning or dimensional reduction. This solves the puzzle of the IIB theory in the duality web as shown in the duality diamond (2.1) including F-theory.

In this paper we focus on the $\mathrm{E}_{5}=\mathrm{SO}(5,5)$ F-theory. The $\mathrm{SO}(5,5)$ current algebra is realized by a 10-brane. The spacetime coordinate is the 16-dimensional spinor representation of $\mathrm{SO}(5,5)$ while the worldvolume coordinate is 10-dimensional vector representation of it. 16 is decomposed into $5+10+1$ under GL(5) symmetry where 5, 10, 1 correspond to the 5-dimensional momentum, the M2-brane winding mode, the M5-brane winding mode respectively. This is a generalization of doubling the spacetime coordinate for linear realization of the $\mathrm{O}(D, D)$ T-duality symmetry as $D$ momenta plus $D$ winding modes. We propose two different ways of writing the F-theory 10-brane actions: 1. the Hamiltonian form action and 2. Lagrangian form action.

1. The Hamiltonian form action is based on the $\mathrm{SO}(5,5)$ "G-symmetry" current algebra. The Lagrangian is written in terms of the selfdual and anti-selfdual field strengths, $\stackrel{\circ}{F}_{\mathrm{SD}}{ }^{\mu}$ and $\stackrel{\circ}{F}_{\mathrm{SD}}{ }^{\mu}$ with $\mu=1, \cdots, 16$. The background gauge fields $G_{\mu \nu}$ are coset 
elements of $\mathrm{G} / \mathrm{H}$ where $\mathrm{H}$ is a subgroup of $\mathrm{G}$. The worldvolume index is $\underline{m}=1, \cdots, 10$ and $\gamma_{\underline{m} \mu \nu}$ is the 10-dimensional gamma matrix. We propose the $\mathrm{SO}(5,5)$ symmetric F-theory 10-brane action in curved backgrounds in (4.14) as

$$
\begin{aligned}
I & =\int d \tau d^{10} \sigma L \\
L & =\frac{1}{g} \stackrel{\circ}{F}_{\mathrm{SD}}^{\mu} G_{\mu \nu} \stackrel{\circ}{F}_{\overline{\mathrm{SD}}^{\nu}}^{\nu}-\hat{\lambda} \stackrel{\circ}{{ }_{\overline{\mathrm{SD}}}}
\end{aligned}
$$

where $g, \hat{\lambda}$ and $\lambda^{\underline{m}}$ are Lagrange multipliers.

2. In the Lagrangian formalism the $\mathrm{SO}(5,5) \mathrm{U}$-duality symmetry is enlarged to $\mathrm{SO}(6,6)$ "F-symmetry". The field strength is a 12-dimensional Weyl spinor $\stackrel{\circ}{F}_{\underline{\mu}}$ with $\underline{\mu}=$ $1, \cdots, 32$. The background gauge fields $G \underline{\mu \nu}$ are coset elements of $\mathrm{F} / \mathrm{L}$ where $\mathrm{L}$ is a subgroup of $\mathrm{F}$. The worldvolume index is $\underline{\hat{m}}=1, \cdots, 12$ and $\Sigma \underline{\underline{\hat{m}}}$ is the 12-dimensional gamma matrix with $\left.\sum \frac{\hat{\hat{m}} \hat{n}}{2}=\frac{1}{2} \sum \underline{\underline{\hat{n}}} \sum \underline{\hat{n}}\right]$. We propose the $\mathrm{SO}(6,6)$ symmetric F-theory 10-brane action in curved backgrounds in (4.28) as

$$
\begin{aligned}
I & =\int d^{12} \sigma L \\
L & =e \stackrel{\circ}{F}_{\underline{\mu}} G \underline{\mu \nu} \stackrel{\circ}{F}_{\underline{\nu}}-\frac{1}{2} \lambda_{\underline{\hat{m} \hat{n}}} \stackrel{\circ}{F}_{\underline{\mu}}\left(C \sum \stackrel{\hat{m} \hat{n}}{\mu}\right) \underline{\mu \nu} \stackrel{\circ}{F}_{\underline{\nu}}
\end{aligned}
$$

where $e$ and $\lambda_{\underline{\hat{m} \hat{n}}}$ are Lagrange multipliers.

The organization of the paper is as follows. In section 3 we present complete sets of the $\mathrm{SO}(5,5)$ current algebras in both the $\mathrm{SO}(5,5)$ spinor representation in subsection 3.1 and the GL(5) tensor representation in subsection 3.2. The former reveals the structure of the current algebra such as the bosonic $\kappa$-symmetry, while the later gives direct coupling to the 11-dimensional supergravity background. One of the author presented the $\operatorname{SO}(5,5)$ current algebra of the M5-brane [41] obtained from the Pasti-Sorokin-Tonin (PST) M5brane Lagrangian [52]. This $\mathrm{SO}(5,5)$ current algebra is recognized as the F-theory 10brane current algebra by doubling the worldvolume coordinate as shown in (3.21), (3.24) and (3.25). In subsection 4.1 we begin by reviewing the double zweibein method to obtain the worldsheet covariant action [10,11] as a method to overcome the chiral action [53]. By applying this method to F-theory we obtain the $\mathrm{SO}(5,5)$ F-theory 10-brane action in the Hamiltonian formalism in subsection 4.2. We extend it to the $\mathrm{SO}(6,6)$ F-theory 10-brane action in subsection 4.3. In the $\mathrm{SO}(6,6)$ Lagrangian the worldvolume vielbein merges with the spacetime vielbein. In subsection 4.4 we present F-theory 10-brane actions in terms of GL(6) and GL(5) tensors to couple the supergravity background. In section 5 we present an action for a 5 -brane obtained from the F-theory 10 -brane action. 5 worldvolume dimensions are reduced by solving the worldvolume section constraint $\mathcal{V}=\partial^{m} \bar{\partial}_{m}=0$. The obtained action for a M-theory 5-brane is sum of the free kinetic term and bilinears of the selfduality constraint. 


\section{Introduction to F-theory}

We begin by an introduction to "theories" with manifest dualities such as T-theory and Ftheory together with "S-theory" and "M-theory". S-theory is a string theory compactified to $D$-dimensions and M-theory is a brane theory compactified to $(D+1)$-dimensions. "Theories" are defined by current algebras with G-symmetry in the Hamiltonian formalism. The background gauge fields are parameters of cosets $\mathrm{G} / \mathrm{H}$ which are generalization of the $\mathrm{GL}(D) / \mathrm{SO}(D-1,1)$ for the vielbein gauge field of the Einstein gravity. All bosonic component fields are representation of $\mathrm{G}$, while fermionic fields are representation of $\mathrm{H}$. A new duality web given in the diamond diagram in (2.1) [45].

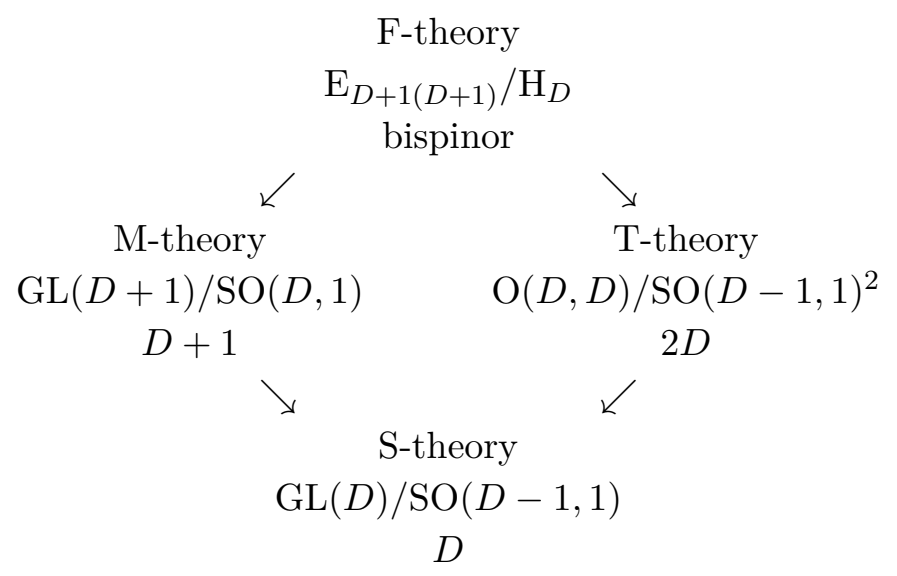

Figure: Diamond diagram

Theories are also defined by worldvolume actions. The Hamiltonian form action is obtained from the G-symmetry current algebra. The spacetime and the brane worldvolume are representations of G-symmetry. The action is written as bilinear of the field strength $F$. There is a gauge symmetry generated by the Gauss law constraint with the gauge parameter $\kappa$. The spacetime coordinate $X_{\sigma}$ plays the gauge field while the auxiliary coordinate $X_{\tau}$ corresponds to $A_{0}$ in the usual gauge theory. The worldvolume coordinate is denoted by $\partial=\frac{\partial}{\partial \sigma}$. We focus on the $D=4 \mathrm{E}_{5}=\mathrm{SO}(5,5)$ G-symmetry case in this paper. Representations of the G-symmetries of theories for the $D=4$ case are summarized in table 1 . The $D=4 \mathrm{~F}$-theory is described by the 10 -brane in the 16 -dimensional spacetime. The 10-dimensional chiral spinor has a bosonic $\kappa$-symmetry-like structure similar to the Green-Schwarz superstring. The $D=4 \mathrm{~F}$-theory in the lightcone-like gauge fixing reduces to the T-theory in the 4-dimensional spacetime.

The G-symmetry is enlarged to F-symmetry in the Lagrangian formulation where the worldvolume Lorentz covariance is manifest. In the usual gauge theory the G-symmetric field strengths correspond to the nonrelativistic electric field and magnetic field while the Fsymmetric field strength corresponds to the Lorentz covariant field strength. The constraint $\mathcal{V}=0$ is a worldvolume section condition. The F-symmetries and representations of theories are summarized as in the table 2 .

The F-symmetry includes the worldvolume symmetry. For example the worldsheet zweibein is in the $\mathrm{SL}(2) / \mathrm{SO}(1,1)$ coset parameter. Symmetry groups for $D=4$ case are summarized as in the table 3 . 


\begin{tabular}{|c|c|c|c|c|c|c|}
\hline theory & G-symmetry & $\begin{array}{c}\text { spacetime } \\
X_{\sigma}\end{array}$ & $\begin{array}{c}\text { spacetime } \\
\text { auxiliary } \\
X_{\tau}\end{array}$ & $\begin{array}{l}\text { world } \\
\text {-volume } \\
\text { } \partial\end{array}$ & $\begin{array}{c}\text { field } \\
\text { strength } \\
F\end{array}$ & $\begin{array}{c}\text { gauge } \\
\text { parameter } \\
\kappa\end{array}$ \\
\hline F- & $\mathrm{SO}(5,5)$ & 16 & $16^{\prime}$ & $1 \oplus 10$ & $16 \oplus 16^{\prime}$ & $16 \oplus 16^{\prime}$ \\
\hline M- & GL(5) & $5 \oplus 10^{\prime} \oplus 1$ & $1 \oplus 10 \oplus 5^{\prime}$ & $1 \oplus 5$ & $1 \oplus 10 \oplus 5^{\prime}$ & $1 \oplus 10 \oplus 5^{\prime}$ \\
\hline T- & $\mathrm{O}(4,4)$ & 8 & 0 & $1 \oplus 1$ & $8 \oplus 8$ & 0 \\
\hline S- & GL(4) & 4 & 0 & $1 \oplus 1$ & $4 \oplus 4$ & 0 \\
\hline
\end{tabular}

Table 1. G-symmetries and representations of theories ( $D=4$ case).

\begin{tabular}{|c|c|c|c|c|c|c|}
\hline theory & F-symmetry & $\begin{array}{c}\text { space } \\
\text {-time } \\
X\end{array}$ & $\begin{array}{c}\text { world } \\
\text {-volume } \\
\partial\end{array}$ & $\begin{array}{c}\text { field } \\
\text { strength } \\
\text { F }\end{array}$ & $\begin{array}{c}\text { gauge } \\
\text { parameter } \\
\kappa\end{array}$ & $\begin{array}{c}\text { cons } \\
\text {-traint } \\
\mathcal{V}=0\end{array}$ \\
\hline F- & $\mathrm{SO}(6,6)$ & $32^{\prime}$ & 12 & 32 & 32 & 1 \\
\hline M- & GL(6) & $6 \oplus 20 \oplus 6^{\prime}$ & $6 \oplus 6^{\prime}$ & $1 \oplus 15 \oplus 15^{\prime} \oplus 1$ & $1 \oplus 15 \oplus 15^{\prime} \oplus 1$ & 1 \\
\hline T- & $\mathrm{O}(4,4) \mathrm{SL}(2)$ & $(8,1)$ & $(1,2)$ & $(8,2)$ & 0 & 0 \\
\hline S- & GL(4)SL(2) & $(4,1)$ & $(1,2)$ & $(4,2)$ & 0 & 0 \\
\hline
\end{tabular}

Table 2. F-symmetries and representations of theories ( $D=4$ case).

\begin{tabular}{c|cccc}
\hline theory & F-symmetry & G-symmetry & L-symmetry & H-symmetry \\
\hline F-theory & $\mathrm{SO}(6,6)$ & $\mathrm{SO}(5,5)$ & $\mathrm{SO}(6 ; \mathbb{C})$ & $\mathrm{SO}(5 ; \mathbb{C})$ \\
M-theory & $\mathrm{GL}(6)$ & $\mathrm{GL}(5)$ & $\mathrm{SO}(4,2)$ & $\mathrm{SO}(4,1)$ \\
T-theory & $\mathrm{O}(4,4) \mathrm{SL}(2)$ & $\mathrm{O}(4,4)$ & $\mathrm{SO}(3 ; 1)^{2} \mathrm{SO}(1,1)$ & $\mathrm{SO}(3,1)^{2}$ \\
S-theory & $\mathrm{GL}(4) \mathrm{SL}(2)$ & $\mathrm{GL}(4)$ & $\mathrm{SO}(3,1) \mathrm{SO}(1,1)$ & $\mathrm{SO}(3,1)$ \\
\hline
\end{tabular}

Table 3. Symmetries of theories ( $D=4$ case).

The spacetime and the worldvolume vielbeins are elements of the coset $\mathrm{F} / \mathrm{L}$. The number of dimensions of the coset $\mathrm{F} / \mathrm{L}$ is larger than the one of $\mathrm{G} / \mathrm{H}$ by the number of Lagrange multipliers of Virasoro constraints for the $p$-brane as

$$
\operatorname{dim}\left(\frac{\mathrm{F}}{\mathrm{L}}\right)=\operatorname{dim}\left(\frac{\mathrm{G}}{\mathrm{H}}\right)+p+1 .
$$

Coset groups and the $\mathrm{G} / \mathrm{H}$ background gauge fields for the $D=4$ theories are summarized as the table 4 .

F-theory reduces to T, M, S-theories by reducing the spacetime dimensions or the worldvolume dimensions by the dimensional reduction or the section condition. In this paper we reduce from the F-theory 10-brane to the M-theory 5-brane with preserving the $\mathrm{SO}(5,5) \mathrm{G}$-symmetry, then the obtained M-theory 5-brane couple to $\mathrm{SO}(5,5)$ background gauge fields. 


\begin{tabular}{c|ccc}
\hline theory & $\operatorname{dim}\left(\frac{\mathrm{F}}{\mathrm{L}}\right)$ & $\operatorname{dim}\left(\frac{\mathrm{G}}{\mathrm{H}}\right)$ & $\frac{\mathrm{G}}{\mathrm{H}}$ gauge fields $\quad(m=0,1, \cdots, 3)$ \\
\hline F-theory & 36 & 25 & $g_{m n}, B_{m n}, C_{[\mathrm{RR}]}$ \\
M-theory & 21 & 15 & $g_{\hat{m} \hat{n}}(\hat{m}=0,1, \cdots, 4)$ \\
T-theory & 18 & 16 & $g_{m n}, B_{m n}$ \\
S-theory & 12 & 10 & $g_{m n}$ \\
\hline
\end{tabular}

Table 4. Gauge fields ( $D=4$ case).

\section{$3 \mathrm{SO}(5,5)$ current algebra}

The $D=4$ F-theory manifests the $\mathrm{E}_{5}=\mathrm{SO}(5,5)$ G-symmetry. This theory reduces to the type II superstring theories in the 4 dimensional spacetime. The type II superstring theories in 10 dimensions have 32 supersymmetries. The supercharges have the $\mathrm{H}$-symmetry index, $\mathrm{SO}(5 ; \mathbb{C})=\operatorname{Sp}(4 ; \mathbb{C})$ index $\mathcal{A}, \dot{\mathcal{A}}=1, \cdots, 4$, and the internal space index, $\mathrm{SU}(4)$ index $\mathcal{A}^{\prime}=$ $1, \cdots, 4$. Supercharges are $\mathcal{Q}_{\mathcal{A} \mathcal{A}^{\prime}}, \overline{\mathcal{Q}}_{\dot{\mathcal{A}}} \mathcal{A}^{\prime}$ while translation charges are $\mathcal{P}_{\mathcal{A} \dot{\mathcal{B}}}, \mho_{[\mathcal{A B}]\left[\mathcal{A}^{\prime} \mathcal{B}^{\prime}\right]}$ and $\bar{\mho}_{[\dot{\mathcal{A}} \mathcal{B}]}\left[\mathcal{A}^{\prime} \mathcal{B}^{\prime}\right]$. The superalgebra in the $D=4$ F-theory is given as follows.

$$
\begin{aligned}
\left\{\mathcal{Q}_{\mathcal{A} \mathcal{A}^{\prime}}, \overline{\mathcal{Q}}_{\dot{\mathcal{B}}}{ }^{\mathcal{B}^{\prime}}\right\}=\delta_{\mathcal{A}^{\prime}}^{\mathcal{B}^{\prime}} \mathcal{P}_{\mathcal{A} \dot{\mathcal{B}}} \\
\left\{\mathcal{Q}_{\mathcal{A} \mathcal{A}^{\prime}}, \mathcal{Q}_{\mathcal{B B}^{\prime}}\right\}=\mho_{[\mathcal{A B}]\left[\mathcal{A}^{\prime} \mathcal{B}^{\prime}\right]} \\
\left\{\overline{\mathcal{Q}}_{\mathcal{A}^{\prime}} \overline{\mathcal{A}}^{\prime}, \overline{\mathcal{Q}}_{\dot{\mathcal{B}}} \mathcal{B}^{\prime}\right\}=\bar{\mho}_{[\dot{\mathcal{A}} \mathcal{B}]}\left[\mathcal{A}^{\prime} \mathcal{B}^{\prime}\right]
\end{aligned}
$$

The 16 translation operators $\mathcal{P}_{\mathcal{A} \dot{\mathcal{B}}}$ are decomposed into 4 momenta, 4 winding modes (NSNS charges) and 8 RR charges $(1 \oplus 6 \oplus 1$ for type IIA and $4 \oplus 4$ for type IIB) in the type II superstring theories in 4 dimensions. The rest of bosonic operators $\mho_{[\mathcal{A B}]\left[\mathcal{A}^{\prime} \mathcal{B}^{\prime}\right]}, \bar{\mho}_{[\dot{\mathcal{A}} \dot{\mathcal{B}}]}\left[\mathcal{A}^{\prime} \mathcal{B}^{\prime}\right]$ are internal operators which are linear combinations of the 6-dimensional internal space momenta $\Upsilon_{[\tilde{\mathcal{A}} \tilde{\mathcal{B}}]}, \bar{\Upsilon}^{[\tilde{\mathcal{A}} \tilde{\mathcal{B}}]}$ and winding modes $\mathrm{F}_{[\mathcal{A} \mathcal{B}]\left[\mathcal{A}^{\prime} \mathcal{B}^{\prime}\right]}, F_{[\dot{\mathcal{A}} \mathcal{B}]}\left[\mathcal{A}^{\prime} \mathcal{B}^{\prime}\right]$ as follows.

$$
\begin{aligned}
& \mho_{[\mathcal{A B}]\left[\mathcal{A}^{\prime} \mathcal{B}^{\prime}\right]}=C_{\mathcal{A B}} \Upsilon_{\left[\mathcal{A}^{\prime} \mathcal{B}^{\prime}\right]}+\mathrm{F}_{[\mathcal{A B}]\left[\mathcal{A}^{\prime} \mathcal{B}^{\prime}\right]} \\
& \bar{\mho}_{[\dot{\mathcal{A}} \dot{\mathcal{B}}]}\left[\mathcal{A}^{\prime} \mathcal{B}^{\prime}\right]=C_{\dot{\mathcal{A} \dot{B}}} \bar{\Upsilon}^{\left[\mathcal{A}^{\prime} \mathcal{B}\right]}+\mathrm{F}_{[\dot{\mathcal{A}} \dot{\mathcal{B}}]}\left[\mathcal{A}^{\prime} \mathcal{B}^{\prime}\right] \text {. }
\end{aligned}
$$

At first, we present current algebras in the $\mathrm{SO}(5,5)$ spinor representation. Next, we present it in the GL(5) tensor representation, in which reduction to M-theory is straightforward and coupling to the 5-dimensional subspace of the 11-dimensional supergravity background is manifest.

\section{$3.1 \mathrm{SO}(5,5)$ spinor representation}

The $\mathrm{SO}(5,5)$ current in a flat space is the 16-component spinor $\triangleright_{\mu}$ with $\mu=1, \cdots, 16$, while the worldvolume is the 10 -dimensional vector with the worldvolume spacial derivative $\partial^{\underline{m}}$ for $\underline{m}=1, \cdots, 10$. The $\mathrm{SO}(5,5)$ current algebra in a flat space is given by

$$
\left[\triangleright_{\mu}(\sigma), \triangleright_{\nu}(\sigma)\right]=2 i \gamma_{\underline{m} \mu \nu} \partial^{\underline{m}} \delta\left(\sigma-\sigma^{\prime}\right)
$$


with $\partial^{\underline{m}} \delta(\sigma)=\frac{\partial}{\partial \sigma_{\underline{m}}} \delta^{(10)}(\sigma)$. The 10-dimensional $\gamma$ matrix is defined by

$$
\gamma_{(\underline{m} \mid \mu \rho} \gamma^{l \rho \nu} \eta_{\underline{l} \mid \underline{n})}=2 \eta_{\underline{m n}} \delta_{\mu}^{\nu}
$$

with the $\mathrm{SO}(5,5)$ invariant metric $\eta_{\underline{m n}}$. The gamma matrix $\gamma_{\underline{m} \mu \nu}$ is transformed under the $\mathrm{SO}(5,5)$ transformation as

$$
\mathrm{SO}(5,5) \ni M_{\underline{m}}^{\underline{n}}, \hat{M}_{\mu}^{\nu}, \quad\left(\hat{M}_{\mu}^{\sigma} \gamma_{\underline{n} \sigma \rho} \hat{M}_{\nu}^{\rho}\right) M_{\underline{m}}^{\underline{n}}=\gamma_{\underline{m} \mu \nu}, \quad M_{\underline{l}}^{\underline{m}} \eta^{\underline{l k}} M_{\underline{k}}^{\underline{n}}=\eta \underline{m n} .
$$

The current algebra $(3.3)$ is $\mathrm{SO}(5,5)$ covariant with transformations of the spinor current and the vector derivative as

$$
\triangleright_{\mu} \rightarrow \hat{M}_{\mu}^{\nu} \triangleright_{\nu}, \quad \partial_{\underline{m}} \rightarrow M_{\underline{m}}^{\underline{n}} \partial_{\underline{n}} .
$$

The 16-dimensional SO $(5,5)$ spinor coordinate $X^{\mu}$ and its canonical conjugate $P_{\mu}$ are given by

$$
\left[P_{\mu}(\sigma), X^{\nu}\left(\sigma^{\prime}\right)\right]=\frac{1}{i} \delta_{\mu}^{\nu} \delta\left(\sigma-\sigma^{\prime}\right) .
$$

The selfdual current $\triangleright_{\mu}$, the anti-selfdual current $\tilde{\triangleright}_{\mu}$ and their algebras are given as

$$
\begin{aligned}
& \left\{\begin{array}{l}
\triangleright_{\mu}=P_{\mu}+\gamma_{\underline{m} \mu \nu} \partial^{\underline{m}} X^{\nu}=P_{\mu}+\not \partial_{\mu \nu} X^{\nu} \\
\tilde{\triangleright}_{\mu}=P_{\mu}-\gamma_{\underline{m} \mu \nu} \partial^{\underline{m}} X^{\nu}=P_{\mu}-\not \partial_{\mu \nu} X^{\nu}
\end{array}\right. \\
& \left\{\begin{array}{l}
{\left[\triangleright_{\mu}(\sigma), \triangleright_{\nu}(\sigma)\right]=2 i \gamma_{\underline{m} \mu \nu} \partial^{\underline{m}} \delta\left(\sigma-\sigma^{\prime}\right)} \\
{\left[\triangleright_{\mu}(\sigma), \tilde{\triangleright}_{\nu}(\sigma)\right]=0} \\
{\left[\tilde{\triangleright}_{\mu}(\sigma), \tilde{\triangleright}_{\nu}(\sigma)\right]=-2 i \gamma_{\underline{m} \mu \nu} \partial^{\underline{m}} \delta\left(\sigma-\sigma^{\prime}\right) .}
\end{array}\right.
\end{aligned}
$$

Under the global $\mathrm{SO}(5,5)$ transformation the canonical coordinates are transformed with use of (3.5) in such a way that currents are transformed as a $\mathrm{SO}(5,5)$ spinor

$$
\left\{\begin{array}{c}
P_{\mu} \rightarrow \hat{M}_{\mu}^{\nu} P_{\nu} \\
\partial_{\underline{\underline{m}}} X^{\mu} \rightarrow M^{-1} \underline{n}^{\underline{m}}\left(\partial^{\underline{n}} X^{\nu}\right) \hat{M}_{\nu}^{-1}{ }^{\mu} \\
\Rightarrow\left(P_{\mu} \pm \gamma_{\underline{m} \mu \rho} \partial^{\underline{m}} X^{\rho}\right) \rightarrow \hat{M}_{\mu}{ }^{\nu}\left(P_{\nu} \pm \gamma_{\underline{m} \mu \rho} \partial^{\underline{m}} X^{\rho}\right) .
\end{array}\right.
$$

The 10-dimensional worldvolume $\sigma_{\underline{m}}$-diffeomorphism is generated by the Virasoro constraint $\mathcal{S}^{\underline{m}}=0$, and the $\tau$-diffeomorphism is generated by $\mathcal{T}$. Closure of the Virasoro algebra requires secondary constraints, $\mathcal{U}^{\mu}=0$ and $\mathcal{V}=0$

$$
\begin{aligned}
\mathcal{S}^{\underline{m}} & =\frac{1}{4} \triangleright_{\mu} \gamma^{\underline{\underline{m}} \mu \nu} \triangleright_{\nu}=0 \\
\mathcal{T} & =\frac{1}{4} \triangleright_{\mu} \hat{\eta}^{\mu \nu} \triangleright_{\nu}=0 \\
\mathcal{U}^{\mu} & =\gamma^{\underline{m} \mu \nu} \triangleright_{\nu} \eta_{\underline{m n}} \partial^{\underline{n}}=(\triangleright \not \partial)^{\mu}=0 \\
\mathcal{V} & =\eta_{\underline{m n}} \partial^{\underline{m}} \partial^{\underline{n}}=0 .
\end{aligned}
$$


The constraints $\mathcal{U}^{\mu}=0$ and $\mathcal{V}=0$ are the Gauss law constraints which generate infinite series of gauge symmetries similar to the $\kappa$-symmetry. For the 16 component spacetime current $\nabla_{\mu}$ and the 10 component worldvolume translation $\partial \underline{m}$ which satisfy (3.3), constraints $\mathcal{U}^{\mu}=\triangleright^{\mu}$ and $\mathcal{V}=\partial^{\underline{m}} \partial_{\underline{m}}$ become first class constraints. $\mathcal{V}=0$ constraint is the $\mathrm{SO}(5,5)$ invariant constraint sectioning the worldvolume. $\mathcal{S}^{\underline{m}}=0$ and $\mathcal{U}^{\mu}=0$ are $\mathrm{SO}(5,5)$ covariant, while the metric $\hat{\eta}^{\mu \nu}$ in $\mathcal{T}$ breaks $\mathrm{SO}(5,5)$ to $\mathrm{SO}(5 ; \mathbb{C})$.

They satisfy the following algebra

$$
\begin{aligned}
{\left[\mathcal{S}^{\underline{m}}(\sigma), \triangleright_{\mu}\left(\sigma^{\prime}\right)\right] } & =i\left[2 \triangleright_{\mu} \partial^{\underline{m}}-\gamma^{\underline{m}}{ }_{\mu \nu} \mathcal{U}^{\nu}\right](\sigma) \delta\left(\sigma-\sigma^{\prime}\right) \\
{\left[\mathcal{T}(\sigma), \triangleright_{\mu}\left(\sigma^{\prime}\right)\right] } & =i \hat{\eta}_{\mu \nu} \mathcal{U}^{\nu}(\sigma) \delta\left(\sigma-\sigma^{\prime}\right)
\end{aligned}
$$

where $\partial^{\underline{m}}$ and $\mathcal{U}^{\mu}$ act on $\delta\left(\sigma-\sigma^{\prime}\right)$. The $\mathrm{SO}(5,5)$ indices are raised and lowered by $\eta_{\underline{m}}$ and $\eta \underline{\underline{m n}}$. It is also noted that $\hat{\eta}^{\mu \rho} \gamma_{\underline{\underline{m} \rho \lambda}} \hat{\eta}^{\lambda \nu}=\eta_{\underline{m n}} \gamma^{\underline{\underline{n}} \mu \nu}$. The set of Virasoro algebras is given by

$$
\begin{aligned}
{\left[\mathcal{S}^{\underline{m}}(\sigma), \mathcal{S}^{\underline{n}}\left(\sigma^{\prime}\right)\right]=} & \left.i\left[2 \mathcal{S}^{(\underline{m}} \partial^{\underline{n}}\right)-\frac{1}{2}\left\{\eta^{\underline{m n}}(\triangleright \mathcal{U})-\left(\triangleright \gamma^{\underline{m n}} \mathcal{U}\right)\right\}\right](\sigma) \delta\left(\sigma-\sigma^{\prime}\right) \\
& +i \delta\left(\sigma-\sigma^{\prime}\right)\left[\partial^{(\underline{m}} \mathcal{S}^{\underline{n})}-\frac{1}{2}\left(\mathcal{U} \gamma^{\underline{m n}} \triangleright\right)\right] \\
= & {\left[2\left(\mathcal{S}^{\underline{n}}(\sigma) \partial^{\underline{m}}(\sigma)+\mathcal{S}^{\underline{m}}\left(\sigma^{\prime}\right) \partial^{\underline{n}}(\sigma)\right)\right.} \\
& \left.-\frac{1}{2}\left\{\eta^{\underline{m n}}(\triangleright \mathcal{U})-\left(\triangleright \gamma^{\underline{m n}} \mathcal{U}\right)\right\}(\sigma)\right] \delta\left(\sigma-\sigma^{\prime}\right) \\
& +i \delta\left(\sigma-\sigma^{\prime}\right)\left[\partial^{[\underline{m}} \mathcal{S}^{\underline{n}]}-\frac{1}{2}\left(\mathcal{U} \gamma^{\underline{m n}} \triangleright\right)\right] \\
{\left[\mathcal{S}^{\underline{m}}(\sigma), \mathcal{T}\left(\sigma^{\prime}\right)\right]=} & i\left[4 \mathcal{T} \partial^{\underline{m}}-\frac{1}{2} \triangleright{ }_{\mu} \hat{\eta}^{\mu \nu}\left(\gamma^{\underline{\underline{m}}} \mathcal{U}\right)_{\nu}\right](\sigma) \delta\left(\sigma-\sigma^{\prime}\right) \\
& +i \delta\left(\sigma-\sigma^{\prime}\right)\left[2 \partial^{\underline{m}} \mathcal{T}-\frac{1}{2}\left(\mathcal{U} \gamma^{\underline{m}}\right)_{\mu} \hat{\eta}^{\mu \nu} \triangleright_{\nu}\right] \\
{\left[\mathcal{T}(\sigma), \mathcal{T}\left(\sigma^{\prime}\right)\right]=} & 2 i \mathcal{S}^{\underline{m}} \partial_{\underline{m}}(\sigma) \delta\left(\sigma-\sigma^{\prime}\right)+i \delta\left(\sigma-\sigma^{\prime}\right)\left(\partial_{\underline{m}} \mathcal{S}^{\underline{m}}\right) \\
{\left[\mathcal{S}^{\underline{m}}(\sigma), \mathcal{U}^{\mu}\left(\sigma^{\prime}\right)\right]=} & i\left(\triangleright \gamma^{\underline{m}}\right)^{\mu} \mathcal{V}(\sigma) \delta\left(\sigma-\sigma^{\prime}\right) \\
{\left[\mathcal{T}(\sigma), \mathcal{U}^{\mu}\left(\sigma^{\prime}\right)\right]=} & i\left(\triangleright \hat{\eta}^{\mu}\right)^{\mu}(\sigma) \delta\left(\sigma-\sigma^{\prime}\right) \\
{\left[\mathcal{U}^{\mu}(\sigma), \mathcal{U}^{\nu}\left(\sigma^{\prime}\right)\right]=} & -2 i \not^{\mu \nu} \mathcal{V}(\sigma) \delta\left(\sigma-\sigma^{\prime}\right) .
\end{aligned}
$$

The spacetime coordinate derivative of a function $\Phi(X)$ is given by

$$
\frac{\partial}{\partial X^{\mu}} \Phi(X)=\partial_{\mu} \Phi(X)=i \int d^{10} \sigma^{\prime}\left[\triangleright_{\mu}\left(\sigma^{\prime}\right), \Phi(X(\sigma))\right]
$$

The worldvolume coordinate derivative of a function $\Phi(X(\sigma))$ is given by

$$
\begin{aligned}
\frac{\partial}{\partial \sigma_{\underline{m}}} \Phi(X(\sigma))=\partial^{\underline{m}} \Phi(X(\sigma)) & =i \int d^{10} \sigma^{\prime}\left[\mathcal{S}^{\underline{m}}\left(\sigma^{\prime}\right), \Phi(X(\sigma))\right] \\
& =\frac{1}{2} \triangleright_{\mu} \gamma^{\underline{m}} \mu \nu
\end{aligned}
$$


The $\mathrm{SO}(5,5)$ current algebra in curved backgrounds with torsion $T_{\alpha \beta}{ }^{\gamma}$ is given as follows.

$$
\begin{aligned}
{\left[\triangleright_{\alpha}(\sigma), \triangleright_{\beta}\left(\sigma^{\prime}\right)\right] } & =2 i T_{\alpha \beta}{ }^{\triangleright_{\gamma}} \delta\left(\sigma-\sigma^{\prime}\right)+i \gamma_{\underline{a} \alpha \beta}\left(D^{\underline{a}}(\sigma)-D^{\underline{a}}\left(\sigma^{\prime}\right)\right) \delta\left(\sigma-\sigma^{\prime}\right) \\
T_{\alpha \beta}{ }^{\gamma} & =E_{[\alpha \mid}^{\mu}\left(\partial_{\mu} E_{\mid \beta]}^{\nu}\right) E_{\nu}^{\gamma}-\frac{1}{2}\left(E_{[\alpha \mid}^{\mu} \gamma_{\underline{a} \mu \nu} \partial_{\lambda} E_{\mid \beta]}^{\nu}\right) \gamma^{\underline{a} \lambda \rho} E_{\rho}^{\gamma}
\end{aligned}
$$

The curved space current $\triangleright_{\alpha}$, the flat space current $\triangleright_{\mu}$ and the curved space worldvolume derivative $D^{\underline{a}}$, the flat worldvolume derivative $\partial^{\underline{m}}$ are related by the spacetime vielbein $E_{\alpha}{ }^{\mu}$ and the worldvolume vielbein $\mathcal{E}_{\underline{m}} \underline{a}$ as

$$
\triangleright_{\alpha}=E_{\alpha}^{\mu} \triangleright_{\mu}, \quad D^{\underline{a}}=\mathcal{E}_{\underline{m}}^{\underline{a}} \partial^{\underline{m}} .
$$

The spacetime vielbein $E_{\alpha}{ }^{\mu} \in \mathrm{SO}(5,5) / \mathrm{SO}(5 ; C)$ and the worldvolume vielbein relate the curved background indices $\mu, \underline{m}$ and the flat space indices $\alpha, \underline{a}$ as

$$
E_{\alpha}{ }^{\mu} \gamma_{\underline{\underline{m}} \mu \nu} E_{\beta}{ }^{\nu} \mathcal{E}_{\underline{a}}^{\underline{m}}=\gamma_{\underline{a} \alpha \beta}, \quad \mathcal{E}_{\underline{a}}^{\underline{m}} \eta_{\underline{m n}} \mathcal{E}_{\underline{b}}^{\underline{n}}=\eta_{\underline{a b}} .
$$

The gauge transformation of $E_{\alpha}{ }^{\mu}$ is given by

$$
\begin{aligned}
\delta_{\lambda} E_{\alpha}{ }^{\mu} \triangleright_{\mu}(\sigma) & =i \int d \sigma^{\prime}\left[\lambda^{\nu} \triangleright_{\nu}\left(\sigma^{\prime}\right), E_{\alpha}{ }^{\mu} \triangleright_{\mu}(\sigma)\right] \\
\delta_{\lambda} E_{\alpha}{ }^{\mu} & =\lambda^{\nu} \partial_{\nu} E_{\alpha}{ }^{\mu}-E_{\alpha}{ }^{\nu} \partial_{\nu} \lambda^{\mu}+\left(E_{\alpha} \gamma^{\underline{a}} \partial_{\nu} \lambda\right){\gamma_{\underline{a}}}^{\nu \mu} .
\end{aligned}
$$

In curved backgrounds the $\tau$ component of Virasoro constraint $\mathcal{T}=0$ (3.10) is generalized as

$$
\begin{aligned}
\mathcal{T} & =\frac{1}{4} \triangleright_{\mu} G^{\mu \nu} \triangleright_{\nu}=\frac{1}{4} \triangleright_{\alpha} \hat{\eta}^{\alpha \beta} \triangleright_{\beta} \\
G^{\mu \nu} & =E_{\alpha}{ }^{\mu} \hat{\eta}^{\alpha \beta} E_{\beta}{ }^{\nu} .
\end{aligned}
$$

The spacetime gauge field $G^{\mu \nu}$ is parametrized by elements of the coset $\mathrm{SO}(5,5) / \mathrm{SO}(5 ; \mathbb{C})$. Spacial components of Virasoro constraints $\mathcal{S} \underline{\underline{m}}$ in (3.10) is inert in curved backgrounds as

$$
\frac{1}{4} \triangleright_{\alpha} \gamma^{\underline{a} \alpha \beta} \triangleright_{\beta}=\frac{1}{4} \mathcal{E}_{\underline{m}}^{\underline{a}}\left(\triangleright_{\mu} \gamma^{\underline{m} \mu \nu} \triangleright_{\nu}\right)=\mathcal{E}_{\underline{m}} \underline{\underline{a}} \mathcal{S}^{\underline{m}} .
$$

Background independence of the spacial components of Virasoro constraints makes possible to impose as the section conditions on fields. This is the same property with the T-theory.

\subsection{GL(5) tensor representation}

The $\mathrm{SO}(5,5)$ current algebra in the GL(5) tensor representation was obtained in the M5 brane Hamiltonian [41] from the PST action [52]. The M5-brane is a 11-dimensional supergravity solution which is described by the spacetime coordinate $x^{m}(\sigma), m=0,1, \cdots, 10$, the second rank selfdual gauge field $A_{i j}(\sigma), i=1, \cdots, 5$ and their canonical conjugates $p_{m}(\sigma), E^{i j}(\sigma)$. The currents are the vector, the 2-rank tensor corresponding to the M2 brane charge and the 5-rank tensor corresponding to the M5 brane charge. The $\tau$ diffeomorphism constraint $\mathcal{T}=\frac{1}{2} p_{m}{ }^{2}+\cdots=0$ is written in bilinear of currents. On the other hand the 5-dimensional worldvolume diffeomorphism constraints are given as 
$\mathcal{H}_{i}=p_{n} \partial_{i} x^{m}+\frac{1}{2} E^{j_{1} j_{2}} \partial_{[i} A_{\left.j_{1} j_{2}\right]}=0$. Multiplying the pullback matrices $\partial_{i} x^{m}$ and $E^{i j} \partial_{j} x^{m}$ on $\mathcal{H}_{i}=0$ gives bilinears of currents $\mathcal{S}^{m}=\mathcal{S}^{m_{1} \cdots m_{4}}=0$ as Virasoro constraints.

In the 5-dimensional subspace where the 11-dimensional space is compactified on a 5-dimensional torus the currents combine into the 16 dimensional $\mathrm{SO}(5,5)$ spinor representation. The reducible set of Virasoro constraints $\mathcal{S}^{m}=\mathcal{S}^{m_{1} \cdots m_{4}}=0$ become $\mathcal{S}^{m}=\overline{\mathcal{S}}_{m}=0$ with $m=1, \cdots, 5$ which is the $5+\overline{5}$ vector representation of the $\mathrm{SO}(5,5)$. All these constraints $\mathcal{T}, \mathcal{S}^{m}$ and $\overline{\mathcal{S}}_{m}$ satisfy a closed algebra with secondary constraints.

The $\mathrm{SO}(5,5)$ current algebra is written in terms of the 16-dimensional spacetime currents $\triangleright_{M}=\left(\triangleright_{m}, \triangleright^{m_{1} m_{2}}, \bar{\triangleright}\right)$ as the GL(5) decomposition of 16 component $\mathrm{SO}(5,5)$ spinor current $16 \rightarrow 5 \oplus 10 \oplus 1$. The commutator of the spinor currents gives the vector, so the worldvolume is 10-dimensional space with $\partial^{\underline{m}}=\left(\partial^{m}, \bar{\partial}_{m}\right)$ with $\underline{m}=1, \cdots, 10$, and $m=1, \cdots, 5$. The $\mathrm{SO}(5,5)$ current algebra in the $\mathrm{GL}(5)$ tensor representation is given with the 10-dimensional $\gamma$ matrices $\rho_{\underline{l} M N}$ and $\rho^{\underline{\underline{l}} M N}$ based on [41] by

$$
\begin{aligned}
& {\left[\triangleright_{M}(\sigma), \triangleright_{N}\left(\sigma^{\prime}\right)\right]=2 i \rho_{\underline{m} M N} \partial^{\underline{m}} \delta\left(\sigma-\sigma^{\prime}\right)} \\
& \rho_{(\underline{l} \mid M L} \rho^{\underline{n} L N} \eta_{\underline{n} \mid \underline{k})}=\eta_{\underline{l k}} \delta_{M}^{N} .
\end{aligned}
$$

The $\mathrm{O}(5,5)$ invariant metric $\eta_{m n}$ is given by

$$
\eta_{\underline{m n}}={ }_{m}^{m}\left(\begin{array}{cc}
n & n \\
0 & \delta_{m}^{n} \\
\delta_{n}^{m} & 0
\end{array}\right) .
$$

Concrete expression of $\rho_{\underline{l} M N}$ with arbitrary parameters $s^{\underline{l}}=\left(s^{l}, \bar{s}_{l}\right)$ and $s_{\underline{l}}=\eta_{\underline{l m}} s^{\underline{m}}=$ $\left(\bar{s}_{l}, s^{l}\right)$ is given by

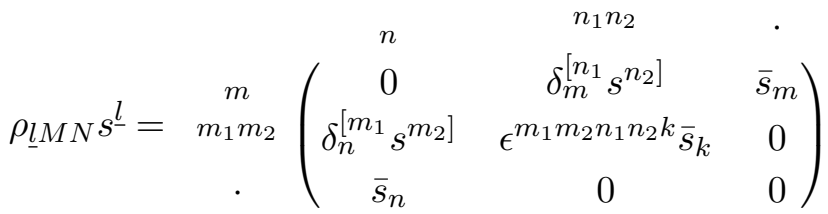

$$
\begin{aligned}
& \rho^{\underline{l} M N} s_{\underline{l}}={ }_{m_{1} m_{2}}^{m}\left(\begin{array}{ccc}
n & n_{1} n_{2} & \cdot \\
\cdot & \delta_{\left[n_{1}\right.}^{m} \bar{s}_{\left.n_{2}\right]} & s^{m} \\
\left.\delta_{\left[m_{1}\right.}^{n} \bar{s}_{\left.m_{2}\right]}\right] & \epsilon_{m_{1} m_{2} n_{1} n_{2} k} s^{k} & 0 \\
s^{n} & 0 & 0
\end{array}\right) .
\end{aligned}
$$

$\rho^{\underline{l} M N}$ satisfies the same $\mathrm{SO}(5,5)$ transformation $(3.5)$. Under the $\mathrm{SO}(5,5)$ transformation it is transformed as

$$
\begin{aligned}
& \mathrm{SO}(5,5) \ni M_{\underline{m}}^{\underline{n}}=\delta_{\underline{m}} \underline{n}+\delta M_{\underline{m}} \underline{\underline{n}}, \hat{M}_{M}^{N}=\delta_{M}{ }^{N}+\delta \hat{M}_{M}{ }^{N} \\
& \left(\rho^{\underline{\underline{l}} M N} s_{\underline{l}}\right) \delta \hat{M}_{M}^{N}+\delta \hat{M}_{M}^{N}\left(\rho^{\underline{\underline{l}} M N} s_{\underline{l}}\right)+\rho^{\underline{l}}{ }^{M N} \delta M_{\underline{l}}^{\underline{\underline{n}}} s_{\underline{n}}=0 \\
& \eta \underline{\underline{m l}} \delta M_{\underline{l}}^{\underline{n}}+\delta M_{\underline{l}} \underline{\underline{m}} \eta \underline{\underline{l n}}=0
\end{aligned}
$$


where infinitesimal $\mathrm{SO}(5,5)$ matircies are given by

$$
\begin{gathered}
\delta \hat{M}_{M}{ }^{N}={ }_{m_{1} m_{2}}^{m}\left(\begin{array}{ccc}
\alpha_{m}{ }^{n}-\frac{\hat{\alpha}}{2} \delta_{m}^{n} & n_{1} n_{2} & \cdot \\
-\tilde{\beta}^{m_{1} m_{2} n} & -\tilde{\gamma}_{m n_{1} n_{2}} & 0 \\
0 & \left.\frac{\hat{\alpha}}{2} \delta_{n_{1}}^{\left[m_{1}\right.} \delta_{n_{2}}^{\left.m_{2}\right]}-\delta_{\left[n_{1}\right.}^{\left[m_{1}\right.} \alpha_{\left.n_{2}\right]} m_{2}\right] & \gamma^{m_{1} m_{2}} \\
\cdot & \beta_{n_{1} n_{2}} & \frac{\hat{\alpha}}{2}
\end{array}\right) \\
\delta M_{\underline{\underline{m}}}{ }^{n}={ }_{m}^{m}\left(\begin{array}{cc}
\alpha_{m}{ }^{n} & \beta_{m n} \\
-\gamma^{m n} & -\alpha_{n}{ }^{m}
\end{array}\right)
\end{gathered}
$$

with $\quad \tilde{\beta}^{m_{1} m_{2} m_{3}}=\frac{1}{2} \epsilon^{m_{1} \cdots m_{5}} \beta_{m_{4} m_{5}}, \quad \tilde{\gamma}_{m_{1} m_{2} m_{3}}=\frac{1}{2} \epsilon_{m_{1} \cdots m_{5}} \gamma^{m_{4} m_{5}}$.

The GL(5) tensor coordinate $X^{M}=\left(X^{m}, X_{m_{1} m_{2}}, \bar{X}\right)$ and its canonical conjugate $P_{M}$ are introduced by

$$
\left[P_{M}(\sigma), X^{N}\left(\sigma^{\prime}\right)\right]=\frac{1}{i} \delta_{M}^{N} \delta\left(\sigma-\sigma^{\prime}\right)
$$

The selfdual and anti-selfdual currents, $\triangleright_{M}$ and $\tilde{\triangleright}_{M}$, together with their current algebras are given by

$$
\begin{aligned}
& \left\{\begin{array}{l}
\triangleright_{M}=P_{M}+\rho_{\underline{m} M N} \partial^{\underline{m}} X^{N} \\
\tilde{\triangleright}_{M}=P_{M}-\rho_{\underline{m} M N} \partial^{\underline{m}} X^{N}
\end{array}\right. \\
& \left\{\begin{array}{l}
{\left[\triangleright_{M}(\sigma), \triangleright_{N}(\sigma)\right]=2 i \rho_{\underline{m} M N} \partial^{\underline{m}} \delta\left(\sigma-\sigma^{\prime}\right)} \\
{\left[\triangleright_{M}(\sigma), \tilde{\triangleright}_{N}(\sigma)\right]=0} \\
{\left[\tilde{\triangleright}_{M}(\sigma), \tilde{\triangleright}_{N}(\sigma)\right]=-2 i \rho_{\underline{m} M N} \partial^{\underline{m}} \delta\left(\sigma-\sigma^{\prime}\right) .}
\end{array}\right.
\end{aligned}
$$

The selfdual currents and their algebra in components are given as

$$
\begin{aligned}
& \left\{\begin{aligned}
\triangleright_{m} & =P_{m}+\partial^{n} X_{m n}+\bar{\partial}_{m} \bar{X} \\
\triangleright^{m_{1} m_{2}} & =P^{m_{1} m_{2}}+\partial^{\left[m_{2}\right.} X^{\left.m_{1}\right]}+\frac{1}{2} \epsilon^{m_{1} \cdots m_{4} l} \bar{\partial}_{l} X_{m_{3} m_{4}} \\
\bar{\triangleright} & =\bar{P}+\bar{\partial}_{m} X^{m}
\end{aligned}\right. \\
& \left\{\begin{aligned}
{\left[\triangleright_{m}(\sigma), \triangleright^{n_{1} n_{2}}\left(\sigma^{\prime}\right)\right] } & =2 i \delta_{m}^{\left[n_{1}\right.} \partial^{\left.n_{2}\right]} \delta\left(\sigma-\sigma^{\prime}\right) \\
{\left[\triangleright_{m}(\sigma), \bar{\triangleright}\left(\sigma^{\prime}\right)\right] } & =2 i \bar{\partial}_{m} \delta\left(\sigma-\sigma^{\prime}\right) \\
{\left[\triangleright^{m_{1} m_{2}}(\sigma), \triangleright^{n_{3} n_{4}}\left(\sigma^{\prime}\right)\right] } & =2 i \epsilon^{m_{1} m_{2} n_{3} n_{4} l} \bar{\partial}_{l} \delta\left(\sigma-\sigma^{\prime}\right) .
\end{aligned}\right.
\end{aligned}
$$


The set of Virasoro constraints in (3.10) is rewritten as

$$
\begin{aligned}
& \mathcal{S}^{\underline{m}}=\frac{1}{4} \triangleright_{M} \rho^{\underline{m} M N} \triangleright_{N}=0 \\
& \mathcal{T}=\frac{1}{4} \triangleright_{M} \hat{\eta}^{M N} \triangleright_{N}=0 \\
& \mathcal{U}^{M}=\rho^{\underline{m}} M N \\
& \eta_{\underline{m n}} \triangleright_{N} \partial^{\underline{n}}=0 \\
& \mathcal{V}=\eta_{\underline{m n}} \partial^{\underline{m}} \partial^{\underline{n}}=0
\end{aligned}
$$

with the $\mathrm{SO}(5 ; \mathbb{C})$ invariant metric

$$
\hat{\eta}^{M N}=m_{m_{1} m_{2}}^{m}\left(\begin{array}{ccc}
\eta^{m n} & n_{1} n_{2} & \cdot \\
0 & \eta_{n_{1}\left[m_{1}\right.} \eta_{\left.m_{2}\right] n_{2}} & 0 \\
0 & 0 & 1
\end{array}\right)
$$

The Virasoro constraints in components are given by

$$
\begin{aligned}
& \left\{\begin{aligned}
\mathcal{S}^{m} & =\frac{1}{2} \triangleright_{n} \triangleright^{n m}=0 \\
\overline{\mathcal{S}}_{m} & =\frac{1}{2}\left[\triangleright_{m} \triangleright \bar{\triangleright}+\frac{1}{8} \epsilon_{m m_{1} \cdots m_{4}} \triangleright^{m_{1} m_{2}} \triangleright^{m_{3} m_{4}}\right]=0 \\
\mathcal{T} & =\frac{1}{4}\left[\triangleright_{m} \eta^{m n} \triangleright_{n}+\frac{1}{2} \triangleright^{m_{1} m_{2}} \eta_{m_{1} n_{1}} \eta_{m_{2} n_{2}} \triangleright^{n_{1} n_{2}}+\bar{\triangleright}^{2}\right]=0 .
\end{aligned}\right. \\
& \left\{\begin{aligned}
\mathcal{U}^{m} & =\bar{\triangleright} \partial^{m}+\triangleright^{m l} \bar{\partial}_{l}=0 \\
\mathcal{U}_{m_{1} m_{2}} & =\triangleright_{\left[m_{1}\right.} \bar{\partial}_{\left.m_{2}\right]}+\frac{1}{2} \epsilon_{m_{1} \cdots m_{5}} \triangleright^{m_{3} m_{4}} \partial^{m_{5}}=0 \\
\overline{\mathcal{U}} & =\triangleright_{m} \partial^{m}=0 \\
\mathcal{V} & =2 \partial^{m} \bar{\partial}_{m}=0 .
\end{aligned}\right.
\end{aligned}
$$

The Virasoro constraint $\mathcal{S}^{\underline{m}}$ generates the shift of the worldvolume coordinate on the current $\triangleright_{M}$ as

$$
\left[\mathcal{S}^{\underline{m}}(\sigma), \triangleright_{M}\left(\sigma^{\prime}\right)\right]=i\left[2 \triangleright_{M} \partial^{\underline{m}}-\eta \underline{\underline{m l}}^{\underline{\underline{l}} M N} \mathcal{U}^{N}\right] \delta\left(\sigma-\sigma^{\prime}\right) .
$$

The Virasoro algebra in the GL(5) tensor representation is the same as (3.12) by replacing the $\gamma \underline{\underline{m}} \mu \nu$-matrices with $\rho \underline{\underline{m}} M N$ in (3.23)

$$
\begin{aligned}
{\left[\mathcal{S}^{\underline{m}}(\sigma), \mathcal{S}^{\underline{n}}\left(\sigma^{\prime}\right)\right]=} & i\left[2 \mathcal{S}^{(\underline{m}} \partial^{\underline{n})}-\frac{1}{2}\left\{\eta^{\underline{m n}}(\triangleright \mathcal{U})-\left(\triangleright \rho^{\underline{m n}} \mathcal{U}\right)\right\}\right](\sigma) \delta\left(\sigma-\sigma^{\prime}\right) \\
& +i \delta\left(\sigma-\sigma^{\prime}\right)\left[\partial^{(\underline{m}} \mathcal{S}^{\underline{n})}-\frac{1}{2}\left(\mathcal{U} \rho^{\underline{m n}} \triangleright\right)\right] \\
{\left[\mathcal{S}^{\underline{m}}(\sigma), \mathcal{T}\left(\sigma^{\prime}\right)\right]=} & i\left\{4 \mathcal{T} \partial^{\underline{m}}-\frac{1}{2} \triangleright_{M} \hat{\eta}^{M N}\left(\rho^{\underline{m}} \mathcal{U}\right)_{N}\right\}(\sigma) \delta\left(\sigma-\sigma^{\prime}\right) \\
& +i \delta\left(\sigma-\sigma^{\prime}\right)\left\{2 \partial^{\underline{m}} \mathcal{T}-\frac{1}{2}\left(\mathcal{U} \rho^{\underline{m}}\right)_{M} \hat{\eta}^{M N} \triangleright_{N}\right\} \\
{\left[\mathcal{T}(\sigma), \mathcal{T}\left(\sigma^{\prime}\right)\right]=} & 2 i \mathcal{S}^{\underline{m}} \partial_{\underline{m}}(\sigma) \delta\left(\sigma-\sigma^{\prime}\right)+i \delta\left(\sigma-\sigma^{\prime}\right)\left(\partial_{\underline{m}} \mathcal{S}^{\underline{m}}\right)
\end{aligned}
$$




$$
\begin{aligned}
{\left[\mathcal{S}^{\underline{m}}(\sigma), \mathcal{U}^{M}\left(\sigma^{\prime}\right)\right] } & =i\left(\triangleright \rho^{\underline{m}}\right)^{M} \mathcal{V}(\sigma) \delta\left(\sigma-\sigma^{\prime}\right) \\
{\left[\mathcal{T}(\sigma), \mathcal{U}^{M}\left(\sigma^{\prime}\right)\right] } & =i(\triangleright \hat{\eta})^{M} \mathcal{V}(\sigma) \delta\left(\sigma-\sigma^{\prime}\right) \\
{\left[\mathcal{U}^{M}(\sigma), \mathcal{U}^{N}\left(\sigma^{\prime}\right)\right] } & =-2 i \not^{M N} \mathcal{V}(\sigma) \delta\left(\sigma-\sigma^{\prime}\right)
\end{aligned}
$$

with $\left(\rho \frac{m n}{}\right)^{M}{ }_{N}=\frac{1}{2} \rho^{[\underline{m} \mid M L} \rho_{\underline{l} L N} \eta^{\underline{l} \mid \underline{n}]}$. The GL(5) tensor expression of the above relation is as follows.

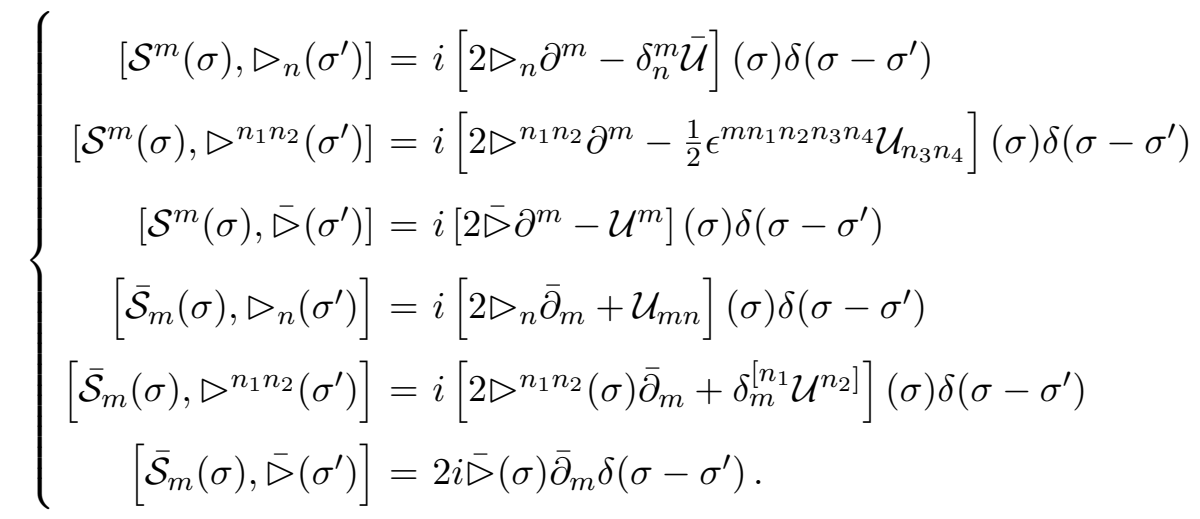

The Virasoro algebra in the GL(5) tensor expression is given by

$$
\begin{aligned}
& {\left[\mathcal{S}^{m}(\sigma), \mathcal{S}^{n}\left(\sigma^{\prime}\right)\right]=i\left[2 \mathcal{S}^{(m} \partial^{n)}-\frac{1}{2}\left(\triangleright^{m n} \overline{\mathcal{U}}-\frac{1}{2} \epsilon^{m n l_{1} l_{2} l_{3}} \triangleright_{l_{1}} \mathcal{U}_{l_{2} l_{3}}\right)\right](\sigma) \delta\left(\sigma-\sigma^{\prime}\right)} \\
& +i \delta\left(\sigma-\sigma^{\prime}\right)\left[\left(\partial^{(m} \mathcal{S}^{n)}\right)-\frac{1}{2}\left(\overline{\mathcal{U}} \triangleright^{m n}-\frac{1}{2} \epsilon^{m n l_{1} l_{2} l_{3}} \mathcal{U}_{l_{1} l_{2}} \triangleright_{l_{3}}\right)\right] \\
& {\left[\mathcal{S}^{m}(\sigma), \overline{\mathcal{S}}_{n}\left(\sigma^{\prime}\right)\right]=i\left[2\left(\mathcal{S}^{m} \bar{\partial}_{n}+\overline{\mathcal{S}}_{n} \partial^{m}\right)-\frac{1}{4} \delta_{n}^{m}\left(\triangleright_{l} \mathcal{U}^{l}+3 \bar{\triangleright} \overline{\mathcal{U}}+\frac{3}{2} \triangleright^{l_{1} l_{2}} \mathcal{U}_{l_{1} l_{2}}\right)\right.} \\
& \left.-\frac{1}{2}\left(\triangleright_{n} \mathcal{U}^{m}-\triangleright^{m l} \mathcal{U}_{n l}\right)\right](\sigma) \delta\left(\sigma-\sigma^{\prime}\right) \\
& +i \delta\left(\sigma-\sigma^{\prime}\right)\left[\left(\partial^{m} \overline{\mathcal{S}}_{n}+\bar{\partial}_{n} \mathcal{S}^{m}\right)+\frac{1}{4} \delta_{n}^{m}\left(\mathcal{U}^{l} \triangleright_{l}-\overline{\mathcal{U}} \triangleright-\frac{1}{2} \mathcal{U}_{l_{1} l_{2}} \triangleright^{l_{1} l_{2}}\right)\right. \\
& \left.-\frac{1}{2}\left(\mathcal{U}^{m} \triangleright_{n}-\mathcal{U}_{n l} \triangleright^{m l}\right)\right] \\
& {\left[\overline{\mathcal{S}}_{m}(\sigma), \overline{\mathcal{S}}_{n}\left(\sigma^{\prime}\right)\right]=i\left[2 \overline{\mathcal{S}}_{(m} \bar{\partial}_{n)}+\frac{1}{2}\left(\bar{\triangleright} \mathcal{U}_{m n}-\frac{1}{2} \epsilon_{m n l_{1} l_{2} l_{3}} \triangleright^{l_{1} l_{2}} \mathcal{U}^{l_{3}}\right)\right](\sigma) \delta\left(\sigma-\sigma^{\prime}\right)} \\
& +i \delta\left(\sigma-\sigma^{\prime}\right)\left[\bar{\partial}_{(m} \overline{\mathcal{S}}_{n)}+\frac{1}{2}\left(\mathcal{U}_{m n} \bar{\triangleright}-\frac{1}{2} \epsilon_{m n l_{1} l_{2} l_{3}} \mathcal{U}^{l_{1}} \triangleright^{l_{2} l_{3}}\right)\right] \\
& {\left[\mathcal{S}^{m}(\sigma), \mathcal{T}\left(\sigma^{\prime}\right)\right]=i\left[4 \mathcal{T} \partial^{m}-\frac{1}{2}\left(\eta^{m k} \triangleright_{k} \overline{\mathcal{U}}+\frac{1}{4} \epsilon^{m m_{1} \cdots m_{4}} \eta_{m_{1} k_{1}} \eta_{m_{2} k_{2}} \triangleright^{k_{1} k_{2}} \mathcal{U}_{m_{3} m_{4}}\right.\right.} \\
& \left.\left.+\bar{\triangleright} \mathcal{U}^{m}\right)\right](\sigma) \delta\left(\sigma-\sigma^{\prime}\right) \\
& +i \delta\left(\sigma-\sigma^{\prime}\right)\left[2 \partial^{m} \mathcal{T}-\frac{1}{2}\left(\eta^{m k} \overline{\mathcal{U}} \triangleright_{k}+\mathcal{U}^{m} \bar{\triangleright}\right.\right. \\
& \left.\left.+\frac{1}{4} \epsilon^{m m_{1} \cdots m_{4}} \eta_{m_{1} k_{1}} \eta_{m_{2} k_{2}} \mathcal{U}_{m_{3} m_{4}} \triangleright^{k_{1} k_{2}}\right)\right](\sigma) \delta\left(\sigma-\sigma^{\prime}\right)
\end{aligned}
$$




$$
\begin{aligned}
{\left[\overline{\mathcal{S}}_{m}(\sigma), \mathcal{T}\left(\sigma^{\prime}\right)\right]=} & i\left[4 \mathcal{T} \bar{\partial}_{m}-\frac{1}{2}\left(\eta^{k n} \triangleright_{k} \mathcal{U}_{n m}+\eta_{k_{1} l} \eta_{k_{2} m} \triangleright^{k_{1} k_{2}} \mathcal{U}^{l}\right)\right](\sigma) \delta\left(\sigma-\sigma^{\prime}\right) \\
& +i \delta\left(\sigma-\sigma^{\prime}\right)\left[2 \bar{\partial}_{m} \mathcal{T}-\frac{1}{2}\left(\eta^{k n} \mathcal{U}_{k m} \triangleright_{n}+\eta_{k_{1} l} \eta_{k_{2} m} \mathcal{U}^{l} \triangleright^{k_{1} k_{2}}\right)\right] \\
{\left[\mathcal{T}(\sigma), \mathcal{T}\left(\sigma^{\prime}\right)\right]=} & 2 i\left(\mathcal{S}^{m} \bar{\partial}_{m}+\overline{\mathcal{S}}_{m} \partial^{m}\right)(\sigma) \delta\left(\sigma-\sigma^{\prime}\right)+i \delta\left(\sigma-\sigma^{\prime}\right)\left(\partial^{m} \overline{\mathcal{S}}_{m}+\bar{\partial}_{m} \mathcal{S}^{m}\right)
\end{aligned}
$$

In order to couple to the 11-dimensional supergravity background the 5-dimensional indices are converted into the 11-dimensional tensor indices as

$$
\begin{array}{ll}
P^{m_{1} \cdots m_{5}}=\epsilon^{m_{1} \cdots m_{5}} \bar{P}, \quad X_{m_{1} \cdots m_{5}}=\epsilon_{m_{1} \cdots m_{5}} \bar{X} \\
\triangleright^{m_{1} \cdots m_{5}}=\epsilon^{m_{1} \cdots m_{5}} \bar{\triangleright}, \quad \tilde{\triangleright}^{m_{1} \cdots m_{5}}=\epsilon^{m_{1} \cdots m_{5}} \bar{\triangleright} \\
\partial^{m_{1} \cdots m_{4}}=\epsilon^{m_{1} \cdots m_{4} l} \bar{\partial}_{l}, \quad \mathcal{S}^{m_{1} \cdots m_{4}}=\epsilon^{m_{1} \cdots m_{4} l} \overline{\mathcal{S}}_{l} .
\end{array}
$$

Currents in the GL(5) tensor representation coupled to the 5-dimensional subspace of the 11-dimensional supergravity background are given as

$$
\begin{aligned}
\triangleright_{A} & =E_{A}{ }^{M} \triangleright_{M} \\
E_{A}{ }^{M} \rho_{\underline{m} M N} E_{B}{ }^{N} \mathcal{E}_{\underline{a}} \underline{\underline{m}} & =\rho_{\underline{a} A B}, \quad \mathcal{E}_{\underline{a}}{ }^{\underline{m}} \eta_{\underline{m n}} \mathcal{E}_{\underline{b}} \underline{\underline{n}}=\eta_{\underline{a b}} \\
E_{A}{ }^{M} & =\left(\begin{array}{ccc}
e_{a}{ }^{m} & e_{a}{ }^{n} C_{n m_{1} m_{2}}^{[3]}-e_{a}{ }^{n} \frac{1}{4 !} C_{n^{\left[m_{1} m_{2}\right.}}^{[3]} C_{\left.m_{3} m_{4} m_{5}\right]}^{[3]} \\
0 & e_{m_{1}}^{a_{1}} e_{m_{2}}{ }^{a_{2}}-\frac{1}{3 !} e_{\left[m_{1}\right.}^{a_{1}} e_{m_{2}}{ }^{a_{2}} C_{\left.m_{3} m_{4} m_{5}\right]}^{[3]} \\
0 & 0 & e_{\left[m_{1}\right.}^{a_{1}} \cdots e_{\left.m_{5}\right]}{ }^{a_{5}}
\end{array}\right) .
\end{aligned}
$$

Under the $\mathrm{SO}(5,5)$ transformation in $\mathcal{T}=0, e_{a}{ }^{m}$ and $C_{m_{1} m_{2} m_{3}}^{[3]}$ are trasnformed fractional linearly. The $\tau$ component of the Virasoro constraints $\mathcal{T}=0$ in a curved background is given by

$$
\begin{aligned}
\mathcal{T} & =\frac{1}{4} \triangleright_{M} G^{M N} \triangleright_{N}=\frac{1}{4} \triangleright_{A} \hat{\eta}^{A B} \triangleright_{B} \\
G^{M N} & =E_{A}{ }^{M} \hat{\eta}^{A B} E_{B}{ }^{N}
\end{aligned}
$$

where $\hat{\eta}^{A B}$ is the same matrix as $\hat{\eta}^{M N}$ in (3.30).

\section{F-theory 10-brane actions}

The F-theory $\mathrm{SO}(5,5)$ current algebras $(3.8)$ or $(3.27)$ are realized on the 10-brane worldvolume which we call F10-brane for short from now on. The Hamiltonian is given by linear combinations of a set of Virasoro constraints (3.10) or (3.29) which are written in terms of the selfdual currents. In order to construct the worldvolume covariant Lagrangian we include the ones for the anti-selfdual currents. At first, we review how to construct the worldsheet covariant action by using the double zweibein method [10,11]. Then we propose actions for the F10-brane with both the $\mathrm{SO}(5,5)$ symmetric Hamiltonian formulation and the $\mathrm{SO}(6,6)$ symmetric Lagrangian formulation. 


\subsection{Double zweibein formulation in T-theory}

The physical current in the T-theory is the selfdual current which is chiral in the doubled space. For the doubled space coordinates $X=(x, y)$ the auxiliary coordinate $y$ is introduced with the selfduality condition; the anti-selfdual current is zero $\partial_{m} x-\epsilon_{m n} \partial^{n} y=0$. We impose the selfduality condition as the first class constraint by squaring of it. The action contains both the selfdual current and the anti-selfdual current leading to the worldsheet covariant action.

The double zweibein formulation of T-theory is given by $\mathrm{O}(D, D)$ current algebras for the selfdual and the anti-selfdual currents, $\nabla_{M}$ and $\tilde{\nabla}_{M}$. These currents are written in terms of the $\mathrm{O}(D, D)$ coordinates, $X^{M}$ and $P_{M}$ with $M=1, \cdots, 2 D$, as

$$
\begin{aligned}
& {\left[P_{M}(\sigma), X^{N}\left(\sigma^{\prime}\right)\right]=\frac{1}{i} \delta_{M}^{N} \partial_{\sigma} \delta\left(\sigma-\sigma^{\prime}\right)} \\
& \left\{\begin{array}{l}
\triangleright_{M}=P_{M}+\partial_{\sigma} X^{N} \eta_{N M} \\
\tilde{\triangleright}_{M}=P_{M}-\partial_{\sigma} X^{N} \eta_{N M}
\end{array}\right. \\
& \left\{\begin{array}{l}
{\left[\triangleright_{M}(\sigma), \triangleright_{N}\left(\sigma^{\prime}\right)\right]=2 i \eta_{M N} \partial_{\sigma} \delta\left(\sigma-\sigma^{\prime}\right)} \\
{\left[\triangleright_{M}(\sigma), \tilde{\triangleright}_{N}\left(\sigma^{\prime}\right)\right]=0} \\
{\left[\tilde{\triangleright}_{M}(\sigma), \tilde{\triangleright}_{N}\left(\sigma^{\prime}\right)\right]=-2 i \eta_{M N} \partial_{\sigma} \delta\left(\sigma-\sigma^{\prime}\right)}
\end{array}\right.
\end{aligned}
$$

with $\partial_{\sigma} \delta(\sigma)=\frac{\partial}{\partial \sigma} \delta^{(1)}(\sigma)$. Virasoro constraints in terms of the selfdual current and the anti-selfdual currents are given by

$$
\left\{\begin{array}{l}
\mathcal{T}=\frac{1}{4} \triangleright_{M} \hat{\eta}^{M N} \triangleright_{N} \\
\mathcal{S}=\frac{1}{4} \triangleright_{M} \eta^{M N} \triangleright_{N}
\end{array}, \quad\left\{\begin{array}{l}
\tilde{\mathcal{T}}=\frac{1}{4} \tilde{\triangleright}_{M} \hat{\eta}^{M N} \tilde{\triangleright}_{N} \\
\tilde{\mathcal{S}}=\frac{1}{4} \tilde{\triangleright}_{M} \eta^{M N} \tilde{\triangleright}_{N}
\end{array}\right.\right.
$$

where $\hat{\eta}^{M N}$ and $\eta^{M N}$ are doubled Minkowski metric and the $\mathrm{O}(D, D)$ invariant metric. The Hamiltonian form action is given by

$$
\begin{aligned}
I & =\int d \tau d \sigma L, \quad L=\dot{X}^{M} P_{M}-H \\
H & =g \mathcal{T}+s \mathcal{S}+\tilde{g} \tilde{\mathcal{T}}+\tilde{s} \tilde{\mathcal{S}} \\
& =\frac{1}{4} \triangleright_{M}(g \hat{\eta}+s \eta)^{M N} \triangleright_{N}+\frac{1}{4} \tilde{\triangleright}_{M}(\tilde{g} \hat{\eta}+\tilde{s} \eta)^{M N} \tilde{\triangleright}_{N}
\end{aligned}
$$

with Lagrange multipliers $g, s, \tilde{g}$, $\tilde{s}$. After the Legendre transformation the obtained Lagrangian is given by

$$
\begin{aligned}
& L=\varphi J_{+}\{(g+\tilde{g}) \hat{\eta}-(s+\tilde{s}) \eta\} J_{-} \\
& \left\{\begin{array}{l}
J_{+}=\dot{X}+(\tilde{g} \hat{\eta}+\tilde{s} \eta) \partial_{\sigma} X \\
J_{-}=\dot{X}-(g \hat{\eta}+s \eta) \partial_{\sigma} X
\end{array}\right. \\
& \varphi=\left[(g+\tilde{g})^{2}-(s+\tilde{s})^{2}\right]^{-1}
\end{aligned}
$$

with $\dot{X}=\partial_{\tau} X$. 
The doubled coordinate has the left moving and the right moving components $X^{M}=$ $\left(X^{\bar{M}}, X \underline{M}\right)$ with $\bar{M}, \underline{M}=1, \cdots, D$. The $D$-dimensional right moving subscript $\underline{M}$ is only used in these two paragraphs, and should not be confused with the enlarged dimension subscript. $D$-dimensional metrics are $\hat{\eta}_{M N}=\operatorname{diag}\left(\eta_{\overline{M N}}, \eta_{M N}\right)$ and the $\mathrm{O}(D, D)$ invariant metric $\eta_{M N}=\operatorname{diag}\left(\eta_{\overline{M N}},-\eta_{\underline{M N}}\right)$. The vielbein field $E_{M}{ }^{A}$ is $\mathrm{O}(D, D)$ gauge field

$$
E_{M}{ }^{A} \eta^{M N} E_{N}{ }^{B}=\eta^{A B} .
$$

With use of the worldsheet doubled zweibeins, $\bar{e}_{a}{ }^{m}$ and $\underline{e}_{a}{ }^{m}$, the Lagrangian (4.3) is rewritten as

$$
\begin{gathered}
L=\frac{1}{\bar{e}} \bar{J}_{+}{ }^{\bar{A}} \eta_{\overline{A B}} \bar{J}_{-}{ }^{\bar{B}}+\underline{1}_{\underline{e}} \underline{J}_{+}{ }^{\underline{A}} \eta_{\underline{A B}} \underline{J}_{-} \underline{\underline{B}} \\
\left\{\begin{array}{l}
\bar{J}_{a}{ }^{\bar{A}}=\bar{e}_{a}{ }^{m} \partial_{m} X^{M} E_{M} \bar{A}^{\bar{A}} \\
\underline{J}_{a}{ }^{\underline{A}}=\underline{e}_{a}{ }^{m} \partial_{m} X^{M} E_{M} \underline{A}
\end{array}\right. \\
e_{a}{ }^{m}=\left(\begin{array}{c}
e_{-}{ }^{\tau} e_{-}{ }^{\sigma} \\
e_{+}{ }^{\tau} e_{+}{ }^{\sigma}
\end{array}\right), \quad \bar{e}_{a}{ }^{m}=\left(\begin{array}{cc}
1 & -(g+s) \\
1 & \tilde{g}+\tilde{s}
\end{array}\right), \quad \underline{e}_{a}{ }^{m}=\left(\begin{array}{cc}
1 & -(g-s) \\
1 & \tilde{g}-\tilde{s}
\end{array}\right)
\end{gathered}
$$

with $\bar{e}=\operatorname{det} \bar{e}_{a}{ }^{m}$ and $\underline{e}=\operatorname{det} \underline{e}_{a}{ }^{m}$.

The Lagrangian with the usual single worldvolume zweibein $e_{a}{ }^{m}$ is given by

$$
\begin{aligned}
& L=\frac{1}{e}\left[J_{+}{ }^{\bar{A}} \eta_{\overline{A B}} J_{-}{ }^{\bar{B}}+J_{+} \underline{\underline{A}} \eta_{\underline{A B}} J_{-\underline{B}}-\lambda_{+} J_{-}{ }^{\bar{A}} \eta_{\overline{A B}} J_{-}{ }^{\bar{B}}-\lambda_{-} J_{+}{ }^{\underline{A}} \eta_{\underline{A B}} J_{+} \underline{B}\right] \\
&\left\{\begin{array}{l}
J_{a}{ }^{\bar{A}}=e_{a}{ }^{m} \partial_{m} X^{M} E_{M} \bar{A} \\
J_{a} \underline{\underline{A}}=e_{a}{ }^{m} \partial_{m} X^{M} E_{M} \underline{\underline{A}}
\end{array}\right. \\
& e_{a}{ }^{m}=\left(\begin{array}{cc}
1 & -(g+s) \\
1 & g-s
\end{array}\right) \\
& \lambda_{ \pm}=\varphi\left\{-(s+\tilde{s} \mp g)^{2}+\tilde{g}^{2}\right\}
\end{aligned}
$$

with $e=\operatorname{det} e_{a}^{m}=2 g$.

It is useful to give the T-theory Lagrangian in terms of the selfdual and the anti-selfdual currents where the anti-selfdual current is the selfduality constraint.

$$
\begin{aligned}
& L=\frac{1}{g} \stackrel{\circ}{J}_{\mathrm{SD}}^{M} G_{M N} \stackrel{\circ}{J}_{\overline{\mathrm{SD}}^{N}}^{N}-\hat{\lambda} \stackrel{\circ}{J}_{\overline{\mathrm{SD}}^{M}}^{M} G_{M N} \stackrel{\circ}{J}_{\overline{\mathrm{SD}}^{N}}^{N}-\lambda \stackrel{\circ}{J}_{\overline{\mathrm{SD}}^{M}}^{M} \eta_{M N} \stackrel{\circ}{J}_{\overline{\mathrm{SD}}^{N}}^{N} \\
& =\frac{1}{g} J_{\mathrm{SD}}^{A} \hat{\eta}_{A B} J_{\overline{\mathrm{SD}}}^{B}-\hat{\lambda} J_{\overline{\mathrm{SD}}}^{A} \hat{\eta}_{A B} J_{\overline{\mathrm{SD}}}^{B}-\lambda J_{\overline{\mathrm{SD}}}^{A} \eta_{A B} J_{\overline{\mathrm{SD}}}^{B} \\
& \left\{\begin{array}{l}
{J_{\mathrm{SD}}}^{A}=E_{M}{ }^{A}{\stackrel{\circ}{J_{\mathrm{SD}}}}^{M} \\
{J_{\overline{\mathrm{SD}}}}^{A}=E_{M}{ }^{A}{\stackrel{\circ}{J_{\overline{\mathrm{SD}}}}}^{M}
\end{array}\right. \\
& \left\{\begin{array}{l}
\stackrel{\circ}{J}_{\mathrm{SD}}^{M}=\dot{X}^{M}+(g \hat{\eta}-s \eta)^{M N} \eta_{N L} \partial_{\sigma} X^{L} \\
\stackrel{\circ}{J} \overline{\mathrm{SD}}^{M}=\dot{X}^{M}-(g \hat{\eta}+s \eta)^{M N} \eta_{N L} \partial_{\sigma} X^{L}
\end{array}\right. \\
& \left\{\begin{array}{l}
\hat{\lambda}=\frac{1}{g}-\varphi(g+\tilde{g}) \\
\lambda=\varphi(s+\tilde{s}) .
\end{array}\right.
\end{aligned}
$$


Bilinears of the anti-selfdual currents relax the selfduality constraint $\stackrel{\circ}{J}_{\overline{S D}^{2}}^{M}=0$ as shown in (D.5). It is the selfduality constraint in curved backgrounds $g^{\tau m} \partial_{m} X^{N} G_{N M}=\epsilon^{\tau m} \partial_{m} X^{N} \eta_{N M}$ with $g^{\tau \tau}=\frac{1}{g}, g^{\tau \sigma}=-\frac{s}{g}$ and $G_{N M}=\hat{\eta}_{N M}$. In this formulation the worldsheet zweibein is not factored out in this Lagrangian.

\section{2 $\mathrm{SO}(5,5)$ Hamiltonian form action}

We apply the double zweibein formulation to construct F10-brane actions. The Hamiltonian is sum of the set of Virasoro constraints $\mathcal{T}=\mathcal{S}^{\underline{m}}=\mathcal{U}^{\mu}=0$ in (3.10) and Virasoro constraints for the anti-selfdual currents $\tilde{\mathcal{T}}=\tilde{\mathcal{S}}^{\underline{m}}=0$ in (4.2) with Lagrange multipliers $g, s_{\underline{m}}, Y_{\mu}, \tilde{g}, \tilde{s}_{\underline{m}}$ respectively. $Y_{\mu}$ plays the role of $A_{0}$ in the usual gauge theory. We begin by the following Hamiltonian form action in the $\mathrm{SO}(5,5)$ spinor representation

$$
\begin{aligned}
I & =\int d \tau d^{10} \sigma L, \quad L=\dot{X}^{\mu} P_{\mu}-H \\
H & =\left(g \mathcal{T}+s_{\underline{m}} \mathcal{S}^{\underline{m}}\right)+\left(\tilde{g} \tilde{\mathcal{T}}+\tilde{s}_{\underline{m}} \tilde{\mathcal{S}}^{\underline{m}}\right)+\mathcal{U}^{\mu} Y_{\mu} \\
& =\frac{1}{4} \triangleright_{\mu}\left(g \hat{\eta}+s_{\underline{m}} \gamma^{\underline{m}}\right)^{\mu \nu} \triangleright_{\nu}+\frac{1}{4} \tilde{\triangleright}_{\mu}\left(\tilde{g} \hat{\eta}+\tilde{s}_{\underline{m}} \gamma^{\underline{m}}\right)^{\mu \nu} \tilde{\triangleright}_{\nu}+\triangleright_{\mu}(\not \partial Y)^{\mu} .
\end{aligned}
$$

The Lagrangian is written in terms of field strengths $\stackrel{\circ}{F}_{ \pm}^{\mu}$ as

$$
\begin{aligned}
& L=\varphi \stackrel{\circ}{F}_{+}{ }^{\mu}\left\{(g+\tilde{g}) \hat{\eta}-(s+\tilde{s})_{\underline{m}} \gamma^{\underline{\underline{m}}}\right\}_{\mu \nu} \stackrel{\circ}{F}_{-}{ }^{\nu}+Y_{\mu} \mathcal{V} X^{\mu} \\
& \left\{\begin{array}{l}
\stackrel{\circ}{F}_{+}{ }^{\mu}=\stackrel{\circ}{F}_{\tau}{ }^{\mu}+\left(\tilde{g} \hat{\eta}+\tilde{s} \underline{\underline{m}} \gamma_{\underline{m}}\right)^{\mu \nu} \stackrel{\circ}{F}_{\sigma \nu} \\
\stackrel{\circ}{F}_{-}{ }^{\mu}=\stackrel{\circ}{F}_{\tau}{ }^{\mu}-\left(g \hat{\eta}+s^{\underline{m}} \gamma_{\underline{m}}\right)^{\mu \nu} \stackrel{\circ}{F}_{\sigma \nu}
\end{array}, \quad\left\{\begin{array}{l}
\stackrel{\circ}{F}_{\tau}{ }^{\mu}=\dot{X}^{\mu}-\not \partial^{\mu \nu} Y_{\nu} \\
\stackrel{\circ}{F}_{\sigma \mu}=\not_{\mu \nu} X^{\nu}
\end{array}\right.\right. \\
& \varphi=\left[(g+\tilde{g})^{2}-(s+\tilde{s})^{\underline{m}}(s+\tilde{s})_{\underline{m}}\right]^{-1}
\end{aligned}
$$

and the $\mathcal{V}=0$ constraint given in (3.10). The field strengths are invariant under the gauge transformation with the gauge parameter $\kappa_{\mu}$ and $\bar{\kappa}^{\mu}$

$$
\delta_{\kappa} X^{\mu}=\not \not^{\mu \nu} \kappa_{\nu}, \quad \delta_{\kappa} Y_{\mu}=\dot{\kappa}_{\mu}+\not \not_{\mu \nu} \bar{\kappa}^{\nu}
$$

by using $\mathcal{V}=0$ constraint. This gauge transformation is generated by the Gauss law constraint as $\delta_{\kappa} X^{\mu}=\left[\int d \sigma \kappa_{\nu} \mathcal{U}^{\nu}, X^{\mu}\right]$. There are gauge symmetries of the gauge symmetry as same as the $\kappa$-symmetry $\delta \kappa=\not \partial \kappa^{[1]}, \delta \kappa^{[1]}=\not \partial \kappa^{[2]}, \cdots$ and $\delta \bar{\kappa}=\not \partial \bar{\kappa}_{[1]}, \delta \bar{\kappa}_{[1]}=\not \partial \bar{\kappa}_{[2]}, \cdots$. The infinite series of gauge symmetries reduce a half of the coordinates. The 10-dimensional worldvolume covariant action requires the auxiliary coordinate $Y_{\mu}$, but it is removed by the bosonic $\kappa$ symmetry in the temporal gauge. In the lightcone-like gauge a half of $X^{\mu}$ is removed, giving the $(4+4)$-dimensional T-theory.

In F-theory currents $F_{ \pm}{ }^{\mu}$ the worldsheet vielbein cannot be extracted from the spacetime vielbein because the $\mathrm{SO}(5,5)$ covariant $\gamma$-matrix $\gamma_{\underline{m} \mu \nu}$ mixes the worldvolume index and the spacetime index unlikely to the $\mathrm{O}(D, D)$ invariant metric $\eta_{M N}$ in the T-theory in (4.4). The first term contains both the selfdual and the anti-selfdual field strengths so it is a free kinetic term. Other terms contain only the anti-selfdual field strength, then they 
are constraints. The F10-brane Lagrangian is further rewritten in terms of the selfdual and the anti-selfdual currents analogously to (4.7) as

$$
\begin{aligned}
& L=\frac{1}{g} \stackrel{\circ}{F}{ }_{\mathrm{SD}}^{\mu} \hat{\eta}_{\mu \nu} \stackrel{\circ}{F} \overline{\mathrm{SD}}^{\nu}-\hat{\lambda} \stackrel{\circ}{{ }_{\mathrm{SD}}}{ }^{\mu} \hat{\eta}_{\mu \nu} \stackrel{\circ}{{ }_{\mathrm{SD}}}{ }^{\nu}-\lambda \stackrel{m}{\stackrel{m}{F}} \overline{\mathrm{SD}}^{\mu} \gamma_{\underline{m} \mu \nu} \stackrel{\circ}{F} \overline{\mathrm{SD}}^{\nu} \\
& \left\{\begin{array}{l}
\stackrel{\circ}{F}_{\mathrm{SD}}{ }^{\mu}=\stackrel{\circ}{F}_{\tau}{ }^{\mu}+\left(g \hat{\eta}-s_{\underline{m}} \gamma^{\underline{m}}\right)^{\mu \nu} \stackrel{\circ}{F}_{\sigma \nu} \\
\stackrel{\circ}{F}_{\overline{\mathrm{SD}}^{\mu}}=\stackrel{\circ}{F}_{\tau}^{\mu}-\left(g \hat{\eta}+s_{\underline{m}} \gamma^{\underline{m}}\right)^{\mu \nu} \stackrel{\circ}{F}_{\sigma \nu}
\end{array}\right. \\
& \left\{\begin{aligned}
\hat{\lambda} & =\frac{1}{g}-\varphi(g+\tilde{g}) \\
\lambda_{\underline{m}} & =\varphi(s+\tilde{s})_{\underline{m}} .
\end{aligned}\right.
\end{aligned}
$$

The selfdual and the anti-selfdual currents in F-theory mixes the worldsheet vielbein and the spacetime vielbein as in (4.7).

In curved background the $\mathrm{SO}(5,5)$ gauge fields $G_{\mu \nu}$ and the $\mathrm{SO}(5,5)$ currents are given with the $\mathrm{SO}(5,5) / \mathrm{SO}(5 ; \mathbb{C})$ vielbein $E_{A}{ }^{M}$ as

$$
G_{\mu \nu}=E_{\mu}^{\alpha} \hat{\eta}_{\alpha \beta} E_{\nu}^{\beta}, F_{ \pm}^{\alpha}=E_{\mu}^{\alpha} \stackrel{\circ}{F}_{ \pm}^{\mu}
$$

The F10-brane Lagrangian in curved background is written as

$$
\begin{aligned}
L & =\varphi \stackrel{\circ}{F}_{+}{ }^{\mu}\left\{(g+\tilde{g}) G_{\mu \nu}-(s+\tilde{s})^{\underline{m}} \gamma_{\underline{m} \mu \nu}\right\} \stackrel{\circ}{F_{-}}{ }^{\nu} \\
& =\varphi F_{+}{ }^{\alpha}\left\{(g+\tilde{g}) \hat{\eta}_{\alpha \beta}-(s+\tilde{s})^{\underline{a}} \gamma_{\underline{a} \alpha \beta}\right\} F_{-}{ }^{\beta}
\end{aligned}
$$

with $\mathrm{SO}(5,5)$ vector parameter $(s+\tilde{s})^{\underline{a}}=(s+\tilde{s})^{\underline{m}} \mathcal{E}_{\underline{m}} \underline{\underline{a}}$, as $(3.5)$.

Then now we propose a Lagrangian for a F10-brane in curved background in terms of the selfdual and the anti-selfdual currents is given as

$$
\begin{aligned}
& L=\frac{1}{g} \stackrel{\circ}{F} \mathrm{SD}^{\mu} G_{\mu \nu} \stackrel{\circ}{F} \overline{\mathrm{SD}}^{\nu}-\hat{\lambda} \stackrel{\circ}{{ }^{\mathrm{SD}}}{ }^{\mu} G_{\mu \nu} \stackrel{\circ}{F} \overline{\mathrm{SD}}^{\nu}-\lambda \stackrel{\underline{m}}{\stackrel{\circ}{F} \overline{\mathrm{SD}}^{\mu} \gamma_{\underline{m} \mu \nu} \stackrel{\circ}{{ }_{\mathrm{SD}}}} \stackrel{\nu}{\nu} \\
& =\frac{1}{g} F_{\mathrm{SD}}^{\alpha} \hat{\eta}_{\alpha \beta} F_{\overline{\mathrm{SD}}}^{\beta}-\hat{\lambda} F_{\overline{\mathrm{SD}}}^{\alpha} \hat{\eta}_{\alpha \beta} F_{\overline{\mathrm{SD}}}^{\beta}-\lambda \underline{a} F_{\overline{\mathrm{SD}}}^{\alpha} \gamma_{\underline{a} \alpha \beta} F_{\overline{\mathrm{SD}}} \beta \\
& \left\{\begin{array}{l}
F_{\mathrm{SD}}^{\alpha}=E_{\mu}{ }^{\alpha} \stackrel{\circ}{F_{\mathrm{SD}}}{ }^{\mu} \\
F_{\overline{\mathrm{SD}}^{\alpha}}=E_{\mu}{ }^{\alpha} \stackrel{\circ}{F}_{\overline{\mathrm{SD}}^{\mu}}^{\mu} .
\end{array}\right.
\end{aligned}
$$

The $\hat{\lambda}$ and $\lambda^{\underline{a}}$ are Lagrange multipliers for selfduality constraints given in (4.11).

\section{3 $\mathrm{SO}(6,6)$ Lagrangian form action}

Next let us consider the $\mathrm{SO}(6,6)$ F-symmetry covariant action. The $\mathrm{SO}(6,6) \gamma$-matrix is given by $64 \times 64$ matrix $\Gamma_{\underline{\underline{a}}}^{\underline{a}}$ with two $32 \times 32$ matrices $\Sigma^{\hat{a}}$ and $\tilde{\Sigma}^{\underline{a}}$ as

$$
\Gamma \underline{\underline{\hat{a}}}=\left(\begin{array}{cc}
0 & \tilde{\Sigma} \underline{\hat{a}} \underline{\underline{\alpha}} \underline{\beta}^{\prime} \\
\Sigma \underline{\hat{a}} \underline{\underline{\alpha} \beta^{\prime}} & 0
\end{array}\right), \quad \underline{\hat{a}}=1, \cdots, 12 .
$$

They satisfy the following algebra with the $\mathrm{SO}(6,6)$ invairant metric $\eta^{\hat{a} \hat{b}}$.

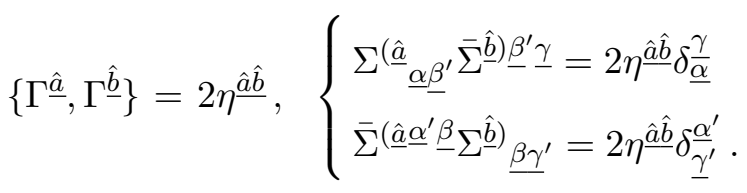


The $\mathrm{SO}(5,5) \gamma$-matrix is embedded as $\underline{\hat{a}}=(+,-, \underline{a}), \quad \underline{a}=(1, \cdots, 10)$

$$
\begin{gathered}
\Sigma_{\underline{\underline{a}} \underline{\underline{\alpha}} \underline{\beta}^{\prime}}: \quad \Sigma^{+}=\left(\begin{array}{cc}
\delta_{\alpha}^{\beta} & 0 \\
0 & 0
\end{array}\right), \quad \Sigma^{-}=\left(\begin{array}{cc}
0 & 0 \\
0 & \delta_{\beta}^{\alpha}
\end{array}\right), \quad \Sigma \underline{a}=\left(\begin{array}{cc}
0 & \gamma^{\underline{a}} \alpha \beta \\
\gamma^{\underline{a}} \alpha \beta & 0
\end{array}\right) \\
\tilde{\Sigma}^{\underline{\hat{a}} \underline{\alpha}^{\prime} \underline{\beta}}: \quad \tilde{\Sigma}^{+}=\left(\begin{array}{cc}
0 & 0 \\
0 & \delta_{\alpha}^{\beta}
\end{array}\right), \quad \tilde{\Sigma}^{-}=\left(\begin{array}{cc}
\delta_{\beta}^{\alpha} & 0 \\
0 & 0
\end{array}\right), \quad \tilde{\Sigma}^{\underline{a}}=-\left(\begin{array}{cc}
0 & \gamma \underline{\gamma}_{\alpha \beta} \\
\gamma^{\underline{a}} \alpha \beta & 0
\end{array}\right) \\
C_{\underline{\alpha} \underline{\beta}}=\left(\begin{array}{cc}
0 & -\delta_{\alpha}^{\beta} \\
\delta_{\beta}^{\alpha} & 0
\end{array}\right), \quad C_{\underline{\alpha}^{\prime} \underline{\beta}^{\prime}}=\left(\begin{array}{cc}
0 & -\delta_{\beta}^{\alpha} \\
\delta_{\alpha}^{\beta} & 0
\end{array}\right)
\end{gathered}
$$

where $\gamma^{\underline{a}}$ is the 10 -dimensional $\gamma$-matrix $\left.\gamma^{(\underline{a}} \alpha \beta \gamma^{\underline{b}}\right) \beta \gamma=2 \eta^{\underline{a b}} \delta_{\alpha}^{\gamma}$ with $\gamma^{\underline{a} \alpha \beta}=\gamma^{\underline{a} \beta \alpha}$. The $\mathrm{SO}(6,6)$ generators are decomposed into the $\mathrm{SO}(5,5)$ dilatation, transformation and rotation as

$$
\begin{aligned}
& \Gamma \frac{\hat{a} \hat{b}}{\underline{\underline{b}}} \underline{\underline{\beta}}=\frac{1}{2}\left(\Sigma^{[\underline{\hat{a}}} \Sigma^{\hat{b}]}\right) \underline{\alpha}^{\underline{\beta}} \equiv \Sigma^{\underline{\hat{a}} \underline{\hat{b}}} \underline{\alpha}^{\underline{\beta}} \\
& \Sigma^{+-} \underline{\alpha}^{\underline{\beta}}=\left(\begin{array}{cc}
\delta_{\alpha}{ }^{\beta} & 0 \\
0 & -\delta^{\alpha}{ }_{\beta}
\end{array}\right), \quad \Sigma^{\underline{a b}} \underline{\alpha}^{\underline{\beta}}=-\left(\begin{array}{cc}
\gamma \underline{\underline{a b}} \alpha^{\beta} & 0 \\
0 & \gamma \underline{a b} \alpha_{\beta}
\end{array}\right) \\
& \Sigma^{-\underline{a}} \underline{\alpha}^{\underline{\beta}}=\left(\begin{array}{cc}
0 & 0 \\
-\gamma^{\underline{a}} \alpha \beta & 0
\end{array}\right), \quad \Sigma^{+\underline{a}} \underline{\alpha}^{\underline{\beta}}=\left(\begin{array}{cc}
0 & -\gamma \underline{a} \\
0 & 0
\end{array}\right) .
\end{aligned}
$$

We use $\Sigma$ 's as the 12-dimensional Weyl-spinors. It is also convenient to have explicit notation of matrices

$$
\begin{aligned}
& \left(C \Gamma^{\underline{\hat{a} \hat{b}}}\right)^{\underline{\alpha} \underline{\beta}}=\frac{1}{2}\left(C \Sigma^{[\underline{\hat{a}}} \tilde{\Sigma}^{\hat{b}]}\right)^{\underline{\alpha} \underline{\beta}} \\
& \left(C \Sigma^{+-}\right)^{\underline{\alpha} \underline{\beta}}=\left(\begin{array}{cc}
0 & -\delta_{\beta}^{\alpha} \\
-\delta_{\alpha}^{\beta} & 0
\end{array}\right), \quad\left(C \Sigma^{\underline{a b}}\right)^{\underline{\alpha} \underline{\beta}}=\left(\begin{array}{cc}
0 & -\gamma \underline{\underline{a b}}_{\beta} \\
\gamma \underline{\underline{a b}} \alpha^{\beta} & 0
\end{array}\right) \\
& \left(C \Sigma^{-\underline{a}}\right)^{\underline{\alpha} \underline{\beta}}=\left(\begin{array}{cc}
-\gamma \underline{\underline{a}} \alpha \beta & 0 \\
0 & 0
\end{array}\right), \quad\left(C \Sigma^{+\underline{a}}\right)^{\underline{\alpha} \underline{\beta}}=\left(\begin{array}{cc}
0 & 0 \\
0 & \gamma \underline{\underline{a}} \alpha \beta
\end{array}\right) .
\end{aligned}
$$

Let's rewrite the $\mathrm{SO}(5,5)$ covariant action $(4.14)$ in a $\mathrm{SO}(6,6)$ covariant way. Two 16-component Majorana-Weyl 10-dimensional spinors $X^{\mu}$ and $Y_{\mu}$ are embedded into a 32-component Majorana-Weyl 12-dimensional spinor

$$
Z^{\underline{\alpha^{\prime}}}=\left(\begin{array}{c}
-Y_{\alpha} \\
X^{\alpha}
\end{array}\right), \underline{\alpha}^{\prime}=1, \cdots, 32 .
$$

The $\mathrm{SO}(6,6)$ covariant field strength in a flat background is given by

$$
\begin{aligned}
& \stackrel{\circ}{F}_{\underline{\alpha}}=\Sigma_{\underline{\underline{\alpha}} \underline{\beta}^{\prime}}^{\partial_{\underline{\hat{a}}}} Z^{\underline{\beta}^{\prime}}=\left(\begin{array}{c}
F_{\sigma \alpha} \alpha \\
F_{\tau}{ }^{\alpha}
\end{array}\right), \quad \underline{\alpha}=1, \cdots, 32 \\
& =\left(\begin{array}{cc}
\delta_{\alpha}^{\beta} \partial^{-} & \not_{\alpha \beta} \\
\not^{\alpha \beta} & \delta_{\beta}^{\alpha} \partial^{+}
\end{array}\right)\left(\begin{array}{c}
-Y_{\beta} \\
X^{\beta}
\end{array}\right)=\left(\begin{array}{c}
(\not \partial X)_{\alpha}-\partial^{-} Y_{\alpha} \\
\partial^{+} X^{\alpha}-(\not \partial Y)^{\alpha}
\end{array}\right) .
\end{aligned}
$$

The field strengths are invariant under the gauge symmetry

$$
\delta_{\kappa} Z^{\underline{\alpha}^{\prime}}=\Sigma \underline{\hat{a}}^{\prime} \underline{\beta}^{\prime} \partial_{\underline{\hat{a}}} \kappa_{\underline{\beta}} .
$$


The $\mathcal{V}=0$ constraint in $(3.10)$ is enlarged $\mathrm{SO}(6,6)$ covariantly.

$$
\begin{aligned}
& \mathcal{V}=\eta_{\underline{m n}} \partial^{\underline{m}} \partial^{\underline{n}}=0 \text { with } \partial^{-}=0 \\
& \rightarrow \hat{\mathcal{V}}={\stackrel{\circ}{\eta_{\hat{m} \hat{n}}}}^{\partial^{\hat{m}}} \partial^{\hat{\hat{n}}}=\eta_{\underline{m n}} \partial^{\underline{m}} \partial^{\underline{n}}+2 \partial^{+} \partial^{-}=0 .
\end{aligned}
$$

We propose a F10-brane Lagrangian in a flat space with the worldvolume vielbein by rewriting the $\mathrm{SO}(5,5)$ covariant action (4.14) with the $\mathrm{SO}(6,6)$ field strength (4.21) with the $32 \times 32$ matrix metric $\stackrel{\circ}{\eta}^{\underline{\alpha}}-$ as

$$
\begin{aligned}
& L=e F_{\underline{\alpha}} \eta^{\underline{\alpha}} \underline{\underline{\beta}} F_{\underline{\beta}}-\frac{1}{2} \lambda_{\underline{\underline{\hat{a}} \hat{b}}} F_{\underline{\alpha}}\left(C \Sigma^{\underline{\hat{a} \hat{b}}}\right)^{\underline{\alpha} \underline{\beta}} F_{\underline{\beta}} \\
& =e \stackrel{\circ}{F}_{\underline{\alpha}}\left(E^{T} \stackrel{\circ}{\eta} E\right)^{\underline{\alpha} \beta} \stackrel{\circ}{F}_{\underline{\beta}}-\frac{1}{2} \lambda_{\hat{a} \hat{b}} \stackrel{\circ}{F}_{\underline{\alpha}}\left(E^{T} C \Sigma \Sigma^{\underline{\hat{a}} \hat{b}} E\right)^{\underline{\alpha} \underline{\beta}} \stackrel{\circ}{F}_{\underline{\beta}} \\
& \stackrel{\circ}{\eta}^{\underline{\alpha}} \underline{-}=\left(\begin{array}{cc}
-\hat{\eta}^{\alpha \beta} & 0 \\
0 & \hat{\eta}_{\alpha \beta}
\end{array}\right) .
\end{aligned}
$$

The field strength with the worldvolume vielbein in a flat space $F_{\underline{\alpha}}$ is given by

$$
\begin{aligned}
F_{\underline{\alpha}} & =\exp \left(\frac{1}{2} f_{\underline{a b}} \Sigma^{\underline{a b}}\right) \underline{\alpha}^{\underline{\beta}} \stackrel{\circ}{F}_{\underline{\beta}} \\
\exp \left(\frac{1}{2} f_{\underline{a b}} \Sigma^{\underline{a b}}\right) \underline{\underline{\alpha}} \underline{\underline{\beta}} & =\exp \left(\frac{1}{2} \ln \phi \Sigma^{+-}\right)_{\underline{\alpha}} \underline{\underline{\alpha}} \exp \left(-\psi_{\underline{a}} \Sigma^{-\underline{a}}\right)_{\underline{\gamma}} \underline{\beta} \\
& =\left(\begin{array}{cc}
\sqrt{\phi} \delta_{\alpha}^{\gamma} & 0 \\
0 & \frac{1}{\sqrt{\phi}} \delta_{\gamma}^{\alpha}
\end{array}\right)\left(\begin{array}{cc}
\delta_{\gamma}^{\beta} & 0 \\
\psi_{\underline{a}} \gamma^{\underline{\alpha} \alpha \beta} & \delta_{\beta}^{\gamma}
\end{array}\right)
\end{aligned}
$$

with $f_{+-}=\ln \phi$ and $f_{-\underline{a}}=-\psi_{\underline{a}}$. The Gauss law constraint is derived from the Lagrangian analogously to the usual gauge theory action. Lagrange multipliers, $e, \lambda_{\underline{a} \hat{b}}$, and the worldvolume vielbein fields $\phi, \psi_{\underline{a}}$, in the $\mathrm{SO}(6,6)$ action (4.24) correspond to Lagrange multipliers of Virasoro constraints for selfdual and anti-selfdual parts, $g, s_{\underline{a}}$ and $\tilde{g}, \tilde{s}_{\underline{a}}$, in the $\mathrm{SO}(5,5)$ action $(4.13)$ as

$$
\left\{\begin{array} { c } 
{ e = \sqrt { \varphi g \tilde { g } } } \\
{ \phi = \sqrt { \frac { g \tilde { g } } { \varphi } } \frac { 1 } { g + \tilde { g } } , } \\
{ \psi _ { \underline { a } } = \frac { g \tilde { s } _ { \underline { a } } - \tilde { g } s _ { \underline { a } } } { g + \tilde { g } } }
\end{array} \left\{\begin{array}{l}
\lambda_{+-}=\frac{g-\tilde{g}}{2(g+\tilde{g})} \\
\lambda_{\underline{a} \underline{b}}=\varphi \tilde{s}_{[\underline{a}} s_{\underline{b}]} \\
\lambda_{+\underline{a}}=-\frac{\sqrt{g \tilde{g} \varphi}}{g+\tilde{g}}\left(s_{\underline{a}}+\tilde{s}_{\underline{a}}\right) \\
\lambda_{-\underline{a}}=-\lambda_{+\underline{a}}-\frac{2}{\phi} \lambda_{\underline{a} \underline{b}} \psi^{\underline{b}}
\end{array}\right.\right.
$$

with $\varphi^{-1}=(g+\tilde{g})^{2}-(s+\tilde{s})^{2}$. They are solved inversely as

$$
\left\{\begin{aligned}
g & =\frac{2 e \phi}{1-2 \lambda_{+-}} \\
\tilde{g} & =\frac{2 e \phi}{1+2 \lambda_{+-}} \\
s_{\underline{a}} & =-\psi_{\underline{a}}-\frac{2 \phi}{1-2 \lambda_{+-}} \lambda_{+\underline{a}} \\
\tilde{s}_{\underline{a}} & =\psi_{\underline{a}}-\frac{2 \phi}{1+2 \lambda_{+-}} \lambda_{+\underline{a}} .
\end{aligned}\right.
$$


We finally obtain the $\mathrm{SO}(6,6)$ covariant action for the F10-brane in curved backgrounds

$$
\begin{aligned}
& I=\int d^{12} \sigma L \\
& L=e \stackrel{\circ}{F}_{\underline{\mu}} G \underline{\mu \nu} \stackrel{\circ}{F}_{\underline{\nu}}-\frac{1}{2} \lambda_{\underline{\hat{m} \hat{n}}} \stackrel{\circ}{F}_{\underline{\mu}}\left(C \sum \stackrel{\hat{m} \hat{n}}{\mu}\right) \underline{\mu \nu} \stackrel{\circ}{F}_{\underline{\nu}} \\
& =F_{\underline{\alpha}} \eta^{\circ} \underline{\underline{\beta}} F_{\underline{\beta}}-\frac{1}{2} \lambda_{\underline{\hat{a} \hat{b}}} F_{\underline{\alpha}}\left(C \sum^{\frac{\hat{a} \hat{b}}{b}}\right) \frac{\alpha \beta}{} F_{\underline{\beta}} \\
& G \underline{\mu \nu}=E_{\underline{\alpha}} \underline{\underline{\mu}} \underline{{ }^{\circ}} \frac{\alpha \beta}{\underline{\underline{\beta}}} E_{\underline{\beta}}^{\underline{\nu}}
\end{aligned}
$$

where $\lambda_{\underline{\hat{m} \hat{n}}}$ and $\lambda_{\underline{\hat{a} \hat{b}}}$ are related by the worldvolume vielbein as (4.26). The $\mathrm{SO}(6,6)$ covariant field strength in a curved background $F_{\underline{\alpha}}$ and the one in a flat space $\stackrel{\circ}{F}_{\underline{\mu}}$ are given as

$$
\begin{aligned}
& F_{\underline{\alpha}}=E_{\underline{\alpha}} \underline{\underline{\mu}} \stackrel{\circ}{F}_{\underline{\mu}}=\left(\sum^{\underline{\hat{a}}}\right)_{\alpha \underline{\beta^{\prime}}} \mathcal{E}_{\underline{\hat{a}}} \underline{\underline{\hat{m}}} \partial_{\underline{\hat{\hat{m}}}} Z^{\mu^{\prime}} E_{\underline{\mu}^{\prime}} \underline{\beta}^{\prime} \\
& \stackrel{\circ}{F}_{\underline{\mu}}=\left(\Sigma^{\underline{\hat{m}}}\right)_{\mu \nu^{\prime}} \partial_{\underline{\hat{m}}} Z^{\underline{\nu}^{\prime}} \text {. }
\end{aligned}
$$

The $\mathrm{SO}(6,6)$ vielbein satisfies the following condition where the $\mathrm{SO}(5,5)$ vielbein field is embedded in the $\mathrm{SO}(6,6)$ vielbein field as

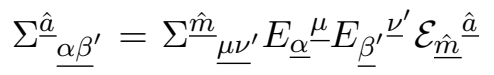

$$
\begin{aligned}
& E_{\underline{\alpha}} \underline{\underline{\mu}}=\exp \left(\frac{1}{2} f_{\underline{a b}} \Sigma^{\underline{a b}}\right) \underline{\alpha}^{\underline{\beta}}\left(\begin{array}{cc}
E_{\beta}{ }^{\mu} & 0 \\
0 & \left(E_{\mu}^{\beta}\right)^{T}
\end{array}\right) \\
& E_{\underline{\alpha}^{\prime}} \underline{\underline{\mu}}^{\prime}=\left(\begin{array}{cc}
\left(E_{\mu}{ }^{\beta}\right)^{T} & 0 \\
0 & E_{\beta}{ }^{\mu}
\end{array}\right) \exp \left(\frac{1}{2} f_{\underline{a b}} \Sigma^{\underline{a b}}\right){\underline{\beta^{\prime}}}^{\prime}{\underline{\alpha^{\prime}}}^{.} .
\end{aligned}
$$

Both the $\mathrm{SO}(5,5)$ spacetime vielbein and the worldvolume vielbein combine into the vielbein field of the $\mathrm{SO}(6,6)$ F-theory. The $\mathrm{SO}(6,6)$ F-theory background is described by the coset $\mathrm{SO}(6,6) / \mathrm{SO}(6 ; \mathbb{C})$ with its dimension $36=25+11$. The number of spacetime vielbein, $\mathrm{SO}(5,5) / \mathrm{SO}(5 ; \mathbb{C})$ fields is 25 , while the number of Virasoro constraints of a $\mathrm{F} 10$ brane is 11 . The $\mathrm{SO}(6,6)$ vielbein is transformed under the $\mathrm{SO}(6,6)$ transformation as

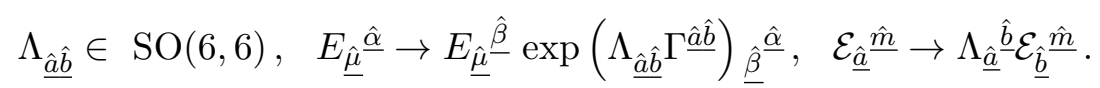




\subsection{GL(6) and GL(5) actions}

In order to construct the perturbative M-theory 5-brane action we rewrite the $\mathrm{SO}(6,6)$ F10-brane action obtained in the previous section (4.28) to the ones with GL(6) and GL(5) symmetries. The $\mathrm{SO}(6,6)$ spinor representations are decomposed into the GL(6) and GL(5) tensor representations as follows:

\section{F-symmetry of F-theory}

$\mathrm{SO}(6,6)$

$$
\left\{\begin{array}{l}
Z^{\underline{\mu}}(32) \\
F_{\underline{\mu}}(32) \\
\partial_{\underline{\hat{m}}}(12)
\end{array}\right.
$$

F-symmetry of M-theory

G-symmetry of F-theory

$$
\begin{aligned}
& \text { GL(6) } \\
& \left\{\begin{aligned}
Z^{\underline{M}}(32) & =Z^{\hat{m}}(6) \oplus \bar{Z}_{\hat{m}}(6) \oplus Z_{\hat{m} \hat{n} \hat{l}}(20) \\
F_{\underline{M}}(32) & =F_{\hat{m} \hat{n}}(15) \oplus F^{\hat{m} \hat{n}}(15) \oplus F(1) \oplus \bar{F}(1) \\
\partial_{\underline{\hat{m}}}(12) & =\partial^{\hat{m}}(6) \oplus \partial_{\hat{m}}(6)
\end{aligned}\right. \\
& \mathrm{SO}(5,5) \\
& \int X^{\mu}(16) \oplus Y_{\mu}(16) \\
& \partial_{m}(5) \oplus \bar{\partial}^{m}(5) \oplus \partial^{+}(1) \oplus \partial^{-}(1) \\
& \text { GL(5) } \\
& \left\{\begin{aligned}
Z^{\hat{m}}(6) & =X^{m}(5) \oplus Y(1) \\
\bar{Z}_{\hat{m}}(6) & =Y_{m}(5) \oplus \bar{X}(1) \\
Z^{\hat{m} \hat{n} \hat{l}}(20) & =X_{m n}(10) \oplus Y^{m n}(10) \\
F_{\hat{m} \hat{n}}(15) & =F_{\tau m n}(10) \oplus F_{\sigma m}(5) \\
F^{\hat{m} \hat{n}}(15) & =F_{\sigma}^{m n}(10) \oplus F_{\tau}^{m}(5) \\
F(1) & =F_{\sigma}(1) \\
\bar{F}(1) & =\bar{F}_{\tau} \\
\partial^{\hat{m}}(6) & =\partial^{m}(5) \oplus \partial^{+}(1) \\
\partial_{\hat{m}}(6) & =\partial_{m}(5) \oplus \partial^{-}(1) .
\end{aligned}\right.
\end{aligned}
$$$$
\left\{F_{\mu}(16) \oplus F^{\mu}(16)\right.
$$

The GL(6) and GL(5) covariant field strengths, denoted by $F_{\underline{M}}$ instead of $\stackrel{\circ}{F}_{\underline{M}}$ for simplicity, and their gauge transformation rules are given as below.

GL(6) field strengths

$$
\begin{aligned}
& F_{\underline{M}}=\left(F^{\hat{m} \hat{n}}, F_{\hat{m} \hat{n}}, F, \bar{F}\right), \quad \hat{m}=1, \cdots, 6 \\
& \left\{\begin{aligned}
F^{\hat{m}_{1} \hat{m}_{2}} & =\partial^{\left[\hat{m}_{1}\right.} Z^{\left.\hat{m}_{2}\right]}+\frac{1}{3 !} \epsilon^{\hat{m}_{1} \cdots \hat{m}_{6}} \partial_{\hat{m}_{3}} Z_{\hat{m}_{4} \cdots \hat{m}_{6}} \\
F_{\hat{m}_{1} \hat{m}_{2}} & =\partial_{\left[\hat{m}_{1}\right.} \bar{Z}_{\left.\hat{m}_{2}\right]}+\partial^{\hat{l}} Z_{\hat{m}_{1} \hat{m}_{2} \hat{l}} \\
F & =\partial_{\hat{m}} Z^{\hat{m}} \\
\bar{F} & =\partial^{\hat{m}} \bar{Z}_{\hat{m}}
\end{aligned}\right.
\end{aligned}
$$


Gauge transformations

$$
\begin{aligned}
& \kappa_{\underline{M}}=\left(\kappa^{\hat{m} \hat{n}}, \kappa_{\hat{m} \hat{n}}, \kappa, \bar{\kappa}\right) \\
& \left\{\begin{aligned}
\delta_{\kappa} Z^{\hat{m}} & =\partial^{\hat{m}} \kappa+\partial_{\hat{n}} \kappa^{\hat{n} \hat{m}} \\
\delta_{\kappa} \bar{Z}_{\hat{m}} & =\partial_{\hat{m}} \bar{\kappa}+\partial^{\hat{n}} \kappa_{\hat{n} \hat{m}} \\
\delta_{\kappa} Z_{\hat{m}_{1} \hat{m}_{2} \hat{m}_{3}} & =\frac{1}{2} \partial_{\left[\hat{m}_{1}\right.} \kappa_{\left.\hat{m}_{2} \hat{m}_{3}\right]}+\frac{1}{2} \epsilon_{\hat{m}_{1} \cdots \hat{m}_{6}} \partial^{\hat{m}_{4}} \kappa^{\hat{m}_{5} \hat{m}_{6}}
\end{aligned}\right.
\end{aligned}
$$

GL(5) field strengths

$$
\begin{aligned}
& F_{\underline{M}}=\left(F_{\tau m n}, F_{\tau}^{m}, \bar{F}_{\tau} ; F_{\sigma}^{m n}, F_{\sigma}, F_{\sigma m}\right), m=1, \cdots, 5 \\
& \left\{\begin{aligned}
F_{\tau} m_{1} m_{2} & =\partial^{+} X_{m_{1} m_{2}}+\partial_{\left[m_{1}\right.} Y_{\left.m_{2}\right]}+\frac{1}{2} \epsilon_{m_{1} \cdots m_{5}} \partial^{m_{3}} Y^{m_{4} m_{5}} \\
F_{\tau}^{m} & =\partial^{+} X^{m}-\partial^{m} Y+\partial_{n} Y^{m n} \\
\bar{F}_{\tau} & =\partial^{+} \bar{X}+\partial^{m} Y_{m} \\
F_{\sigma}^{m_{1} m_{2}} & =\partial^{\left[m_{1}\right.} X^{\left.m_{2}\right]}+\frac{1}{2} \epsilon^{m_{1} \cdots m_{5}} \partial_{m_{3}} X_{m_{4} \cdots m_{5}} \\
F_{\sigma m} & =\partial_{m} X-\partial^{n} X_{m n} \\
F_{\sigma} & =\partial_{m} X^{m}
\end{aligned}\right.
\end{aligned}
$$

Gauge transformations

$$
\begin{aligned}
& \kappa_{\underline{M}}=\left(\kappa^{m_{1} m_{2}}, \kappa_{m}, \kappa ; \kappa_{m_{1} m_{2}}, \kappa^{m}, \bar{\kappa}\right) \\
& \left\{\begin{aligned}
\delta_{\kappa} X^{m} & =\partial_{n} \kappa^{n m}+\partial^{m} \kappa \\
\delta_{\kappa} X_{m_{1} m_{2}} & =\partial_{\left[m_{1}\right.} \kappa_{\left.m_{2}\right]}-\epsilon_{m_{1} \cdots m_{5}} \partial^{m_{3}} \kappa^{m_{4} m_{5}} \\
\delta_{\kappa} \bar{X} & =\partial^{m} \kappa_{m}
\end{aligned}\right. \\
& \left\{\begin{aligned}
\delta_{\kappa} Y_{m} & =-\partial^{+} \kappa_{m}+\partial_{m} \bar{\kappa}+\partial^{n} \kappa_{n m} \\
\delta_{\kappa} Y^{m_{1} m_{2}} & =\partial^{+} \kappa^{m_{1} m_{2}}+\partial^{\left[m_{1}\right.} \kappa^{\left.m_{2}\right]}+\frac{1}{2} \epsilon^{m_{1} \cdots m_{5}} \partial_{m_{3}} \kappa_{m_{4} m_{5}} \\
\delta_{\kappa} \bar{Y} & =\partial^{+} \kappa+\partial_{n} \kappa^{n} .
\end{aligned}\right.
\end{aligned}
$$

$\partial^{+}$stands for $\partial_{\tau}$ obtained from the Lagrangian. Using with the $16 \times 16$ matrix $\rho_{\underline{m} M N}$ for $X^{M}=\left(X^{m}, X_{m n}, \bar{X}\right)$ and $Y_{M}=\left(Y_{m}, Y^{m n}, Y\right)$ in (3.5) the field strengths are written as below.

GL(5) field strengths

$$
\begin{aligned}
& F_{\underline{M}}=\left(F_{\tau}^{M}, F_{\sigma M}\right), \quad M=1, \cdots, 16 \\
& \left\{\begin{array}{l}
F_{\tau}^{M}=\dot{X}^{M}-\rho^{\underline{m}} M N \\
F_{\sigma M}=\rho_{\underline{m} M N} \partial^{\underline{m}} X^{N}
\end{array}\right.
\end{aligned}
$$

Gauge transformations

$$
\begin{aligned}
& \kappa_{\underline{M}}=\left(\kappa_{M}, \kappa^{M}\right) \\
& \left\{\begin{array}{c}
\delta_{\kappa} X^{M}=\rho^{\underline{m} M N} \partial_{\underline{m}} \kappa_{N} \\
\delta_{\kappa} Y_{M}=\dot{\kappa}_{M}+\rho_{\underline{m} M N} \partial^{\underline{m}} \kappa^{N} .
\end{array}\right.
\end{aligned}
$$

$\dot{X}$ stands for $\partial_{\tau} X$ obtained from the Hamiltonian form Lagrangian. 
The GL(6) covariant field strength in curved background $F_{\underline{A}}$ is related to the one in a flat background $\stackrel{\circ}{F}_{\underline{M}}$ with the vielbein field $E_{\underline{A}} \underline{\underline{M}}$ which is a coset element of $\mathrm{SO}(6,6) / \mathrm{SO}(6 ; \mathbb{C})$ as well as the one in (4.28) as

$$
G \frac{M N}{\underline{M}}=E_{\underline{A}} \underline{\underline{M}} \eta^{\circ} \stackrel{\underline{A B}}{ } E_{\underline{B}} \underline{\underline{N}}, \quad F_{\underline{A}}=E_{\underline{A}}{ }^{\underline{M}} \stackrel{\circ}{F}_{\underline{M}} .
$$

The number of degrees of freedom of the parameter of the coset $\mathrm{SO}(6,6) / \mathrm{SO}(6 ; \mathbb{C})$ is $36=25+10+1: 25$ is the number of degrees of freedom of the metric and the 3 -form gauge field in 5 dimensions. $10+1$ is the number of worldvolume dimensions constrained by $\mathcal{V}=0,12-1$. The GL(6) covariant F10-brane action in a curved background is obtained from the $\mathrm{SO}(6,6)$ covariant F10-brane action (4.28) in terms of 32-component field strength $F_{\underline{M}}$ in $(4.32)$ as

$$
\begin{aligned}
& I=\int d^{12} \sigma L \\
& L=e \stackrel{\circ}{F}_{\underline{M}} G^{M N} \stackrel{\circ}{F}_{\underline{N}}+\frac{1}{2} \lambda_{\underline{\hat{n} \hat{n}}} \stackrel{\circ}{F}_{M}(C \Sigma \underline{\underline{\hat{m}} \hat{n}}) \frac{M N}{\stackrel{\circ}{F}^{N}}
\end{aligned}
$$

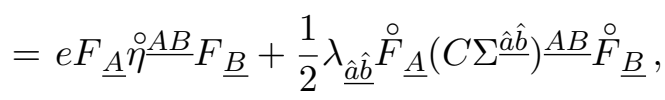

$$
\begin{aligned}
& \stackrel{\circ}{\eta \underline{A B}}=\left(\begin{array}{cc}
\hat{\eta}^{A B} & \\
& -\hat{\eta}_{A B}
\end{array}\right) .
\end{aligned}
$$

The 12-dimensional $\Sigma$ matrices $\left(C \Sigma^{\hat{a} \hat{a}}\right){ }^{A B}$ are the same expression in (4.19) with replacing $\gamma^{\underline{\underline{a}} \alpha \beta} \rightarrow \rho^{\underline{a}} A B, \gamma^{\underline{a}}{ }_{\alpha \beta} \rightarrow \eta^{\underline{a b}} \rho_{\underline{b}} A B$ and $\gamma^{\underline{a b} \alpha}{ }_{\beta} \rightarrow \rho^{[\underline{a} \mid A C} \rho_{\underline{c} C B} \eta^{\underline{c}} \underline{\underline{b}]}$.

The GL(5) tensors directly couple to the 11-dimensional supergravity background. The GL(5) covariant field strength in curved background $F^{A}$ is related to the one in a flat background $\stackrel{\circ}{F}^{M}$ with the vielbein field $E_{A}{ }^{M}$ which is a coset element of $\operatorname{SO}(5,5) / \mathrm{SO}(5 ; \mathbb{C})$ as well as the one in (4.12) as

$$
G_{M N}=E_{M}^{A} \hat{\eta}_{A B} E_{N}^{B}, \quad F^{A}=E_{M}^{A} \stackrel{\circ}{F^{M}}
$$

The Hamiltonian form action given in (4.9) gives the same form with replacing $\gamma \underline{\underline{m}} \mu \nu$ with $\rho^{\underline{m} M N}$. The GL(5) covariant F10-brane in curved background is given as

$$
\begin{aligned}
& I=\int d \tau d^{10} \sigma L \\
& L=\varphi(g+\tilde{g}) \stackrel{\circ}{F}_{+}{ }^{M} G_{M N} \stackrel{\circ}{F}_{-}{ }^{N}-\varphi(s+\tilde{s})^{\underline{l}} \stackrel{\circ}{F}_{+}{ }^{M} \rho_{\underline{l}}{ }_{-}{ }^{\circ} \stackrel{\circ}{F}_{-}{ }^{N} \\
& =\varphi(g+\tilde{g}) F_{+}{ }^{A} \hat{\eta}_{A B} \stackrel{\circ}{F}_{-}^{B}-\varphi(s+\tilde{s})^{\underline{a}} \stackrel{\circ}{F}_{+}^{A} \rho_{\underline{a} A B} F_{-}{ }^{B} \\
& \left\{\begin{array}{l}
\stackrel{\circ}{F}_{+} M=\stackrel{\circ}{F}_{\tau} M+\left(\tilde{g} \hat{\eta}+\tilde{s}_{\underline{m}} \rho^{\underline{m}}\right)^{M N} \stackrel{\circ}{F}_{\sigma N} \\
\stackrel{\circ}{F}_{-}{ }^{M}=\stackrel{\circ}{F}_{\tau} M-\left(g \hat{\eta}+s_{\underline{m}} \rho \underline{m}\right)^{M N} \stackrel{\circ}{F}_{\sigma N} .
\end{array}\right.
\end{aligned}
$$


The GL(5) covariant F10-brane Lagrangian in curved background in terms of the selfdual and the anti-selfdual currents is given by (4.11) as

$$
\begin{aligned}
& L=\frac{1}{g} \stackrel{\circ}{F}{ }_{\mathrm{SD}}^{M} G_{M N} \stackrel{\circ}{F} \overline{\mathrm{SD}}^{N}-\hat{\lambda} \stackrel{\circ}{{ }_{\mathrm{SD}}}{ }^{M} G_{M N} \stackrel{\circ}{F}_{\overline{\mathrm{SD}}^{N}-\lambda \stackrel{m}{F} \stackrel{\circ}{\mathrm{SD}}^{M} \rho_{\underline{m}} M N} \stackrel{\circ}{F}_{\overline{\mathrm{SD}}^{N}}^{N} \\
& =\frac{1}{g} F_{\mathrm{SD}}{ }^{\alpha} \hat{\eta}_{\alpha \beta} F_{\overline{\mathrm{SD}}}{ }^{\beta}-\hat{\lambda} F_{\overline{\mathrm{SD}}}^{\alpha} \hat{\eta}_{\alpha \beta} F_{\overline{\mathrm{SD}}}^{\beta}-\lambda \underline{a} F_{\overline{\mathrm{SD}}}^{\alpha} \gamma_{\underline{a} \alpha \beta} F_{\overline{\mathrm{SD}}}{ }^{\beta} \\
& \left\{\begin{array}{l}
\stackrel{\circ}{F}_{\mathrm{SD}}{ }^{M}=\stackrel{\circ}{F}_{\tau}{ }^{M}+\left(g \hat{\eta}-s_{\underline{m}} \rho^{\underline{m}}\right)^{M N} \stackrel{\circ}{F}_{\sigma N} \\
\stackrel{\circ}{F}_{\overline{\mathrm{SD}}}{ }^{M}=\stackrel{\circ}{F}_{\tau}^{M}-\left(g \hat{\eta}+s_{\underline{m}} \rho^{\underline{m}}\right)^{M N} \stackrel{\circ}{F}_{\sigma N} .
\end{array}\right.
\end{aligned}
$$

In order to couple to the 11-dimensional supergravity background $Y_{m}$ and $\bar{F}$ are rewritten in the 5-dimensional dual as

$$
Y_{m}=\frac{1}{4 !} \epsilon_{m n_{1} \cdots n_{4}} \bar{Y}^{n_{1} \cdots n_{4}}, \quad \bar{F}=\frac{1}{5 !} F_{m_{1} \cdots m_{5}} \epsilon^{m_{1} \cdots m_{5}} .
$$

The background of $\mathrm{SO}(5,5)$ vielbein given in $(3.38)$ is given by the metric $e_{m}{ }^{a}$ and the three form gauge field $C_{m_{1} m_{2} m_{3}}^{[3]}$ in the 5 -dimensional space $m=1, \cdots, 5$. The field strength $\stackrel{\circ}{F}^{M}$ including both $\stackrel{\circ}{F}_{\tau}^{M}$ and $\stackrel{\circ}{F}_{\sigma}{ }^{M}$ in the backgrounds are as follows.

$$
\begin{aligned}
& F^{A}=E_{M}{ }^{A} \stackrel{\circ}{F}^{M} \\
& \left\{\begin{aligned}
F^{a}= & F^{m} e_{m}{ }^{a} \\
F_{a_{1} a_{2}}= & -F^{m} C^{[3]}{ }_{m n_{1} n_{2}} e_{a_{1}}{ }^{n_{1}} e_{a_{2}}{ }^{n_{2}}+F_{m_{1} m_{2}} e_{a_{1}}{ }^{m_{1}} e_{a_{2}}{ }^{m_{2}} \\
F_{a_{1} \cdots a_{5}}= & +\frac{1}{2 \cdot 3 !} F_{m_{1} m_{2}} C^{[3]} m_{3} m_{4} m_{5} e_{\left[a_{1}\right.}{ }^{m_{1}} \cdots e_{\left.a_{5}\right]}{ }^{m_{5}} \\
& +F_{m_{1} \cdots m_{5}} e_{a_{1}}{ }^{m_{1}} \cdots e_{a_{5}}{ }^{m_{5}} .
\end{aligned}\right.
\end{aligned}
$$

\section{Perturbative M-theory 5-brane action}

In order to obtain an action for the perturbative M-theory 5-brane coupled to the 11dimensional supergravity background, we preserve the number of the $\mathrm{SO}(5,5)$ currents. The worldvolume dimensions of F-theory is reduced solving $\mathcal{V}=\partial_{\underline{m}} \eta^{\underline{m n}} \partial_{\underline{n}}=0$ as $\bar{\partial}_{m}=$ $\frac{1}{4 !} \epsilon_{m_{1} \cdots m_{5}} \partial^{m_{2} \cdots m_{5}}=0$ consistently. The 5 -dimensional worldvolume theory is obtained by the following sectioning [51]

$$
\bar{\partial}_{m}=0 \rightarrow \mathcal{V}=2 \partial^{m} \bar{\partial}_{m}=0 .
$$

The selfdual and the anti-selfdual currents for the M5-brane are given as

$$
\begin{gathered}
\left\{\begin{aligned}
\triangleright_{m} & =P_{m}+\partial^{n} X_{m n} \\
\triangleright^{m_{1} m_{2}} & =P^{m_{1} m_{2}}+\partial^{\left[m_{2}\right.} X^{\left.m_{1}\right]} \\
\bar{\triangleright} & =\bar{P}
\end{aligned}\right. \\
\left\{\begin{aligned}
\tilde{\triangleright}_{m} & =P_{m}-\partial^{n} X_{m n} \\
\tilde{\triangleright}^{m_{1} m_{2}} & =P^{m_{1} m_{2}}-\partial^{\left[m_{2}\right.} X^{\left.m_{1}\right]} \\
\overline{\tilde{D}} & =\bar{P} .
\end{aligned}\right.
\end{gathered}
$$


The $\mathrm{SO}(5,5)$ current algebras in $(3.28)$ is reduced to the subalgebra which is the same one for the SL(5) case [40]

$$
\begin{aligned}
\left\{\begin{aligned}
{\left[\triangleright_{m}(\sigma), \triangleright^{n_{1} n_{2}}\left(\sigma^{\prime}\right)\right] } & =2 i \delta_{m}^{\left[n_{1}\right.} \partial^{\left.n_{2}\right]} \delta\left(\sigma-\sigma^{\prime}\right) \\
{\left[\triangleright_{m}(\sigma), \bar{\triangleright}\left(\sigma^{\prime}\right)\right] } & =0 \\
{\left[\triangleright^{m_{1} m_{2}}(\sigma), \triangleright^{n_{3} n_{4}}\left(\sigma^{\prime}\right)\right] } & =0
\end{aligned}\right. \\
\left\{\begin{aligned}
&\left.\tilde{\triangleright}_{\mu}(\sigma), \triangleright_{\nu}\left(\sigma^{\prime}\right)\right]=0 \\
& {\left[\tilde{\triangleright}_{m}(\sigma), \tilde{\triangleright}^{n_{1} n_{2}}\left(\sigma^{\prime}\right)\right] }=-2 i \delta_{m}^{\left[n_{1}\right.} \partial^{\left.n_{2}\right]} \delta\left(\sigma-\sigma^{\prime}\right) \\
& {\left[\tilde{\triangleright}_{m}(\sigma), \overline{D^{\prime}}\left(\sigma^{\prime}\right)\right] }=0 \\
& {\left[\tilde{\triangleright}^{m_{1} m_{2}}(\sigma), \tilde{\triangleright}^{n_{3} n_{4}}\left(\sigma^{\prime}\right)\right] }=0 .
\end{aligned}\right.
\end{aligned}
$$

A set of the Virasoro constraints and the Gauss law constraints in (3.31) and (3.32) are reduced to

$$
\begin{aligned}
& \left\{\begin{aligned}
\mathcal{S}^{m} & =\frac{1}{2} \triangleright_{n} \triangleright^{n m}=0 \\
\overline{\mathcal{S}}_{m} & =\frac{1}{2}\left[\triangleright_{n} \bar{\triangleright}+\frac{1}{8} \epsilon_{m m_{1} \cdots m_{4}} \triangleright^{m_{1} m_{2}} \triangleright^{m_{3} m_{4}}\right]=0 \\
\mathcal{T} & =\frac{1}{2}\left[\triangleright_{m} \eta^{m n} \triangleright_{n}+\frac{1}{2} \triangleright^{m_{1} m_{2}} \eta_{m_{1} n_{2}} \eta_{m_{2} n_{2}} \triangleright^{n_{1} n_{2}}+\bar{\triangleright}^{2}\right]=0
\end{aligned}\right. \\
& \left\{\begin{aligned}
\mathcal{U} & =\triangleright_{m} \partial^{m}=0 \\
\mathcal{U}^{m} & =\bar{\triangleright} \partial^{m}=0 \\
\mathcal{U}_{m_{1} m_{2}} & =\epsilon_{m_{1} \cdots m_{5}} \triangleright^{m_{3} m_{4}} \partial^{m_{5}}=0 \\
\mathcal{V} & =0
\end{aligned}\right.
\end{aligned}
$$

An action for the M-theory 5-brane in curved background is given from the F-theory 10-brane (4.13) by sectioning the worldvolume into 5 dimensions as

$$
\begin{aligned}
I & =\int d \tau d^{5} \sigma L \\
L & =\varphi \stackrel{\circ}{F}_{+}{ }^{M}\left\{(g+\tilde{g}) G_{M N}-\varphi(s+\tilde{s})^{\underline{l}} \rho_{\underline{l}} M N\right\} \stackrel{\circ}{F}_{-}{ }^{N} \\
& =\varphi F_{+}{ }^{A}\left\{(g+\tilde{g}) \hat{\eta}_{A B}-\varphi(s+\tilde{s})^{\underline{a}} \rho_{\underline{a} A B}\right\} F_{-}{ }^{B} .
\end{aligned}
$$

Now let us construct the action for the M-theory 5-brane in terms of the selfdual and the anti-selfdual currents where the anti-selfdual currents are auxiliary introduced to make a free kinetic term. The selfdual (SD) and the anti-selfdual $(\overline{\mathrm{SD}})$ field strengths are 
given by

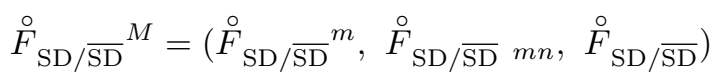

$$
\begin{aligned}
& \left\{\begin{aligned}
\stackrel{\circ}{F}_{\mathrm{SD} / \overline{\mathrm{SD}}^{m}} & =\stackrel{\circ}{F}_{\tau}^{m} \pm\left\{g \eta^{m n} \stackrel{\circ}{F}_{\sigma n}-\left(s_{n} \stackrel{\circ}{F}_{\sigma}^{m n}+\bar{s}^{m} \stackrel{\circ}{F}_{\sigma}\right)\right\} \\
\stackrel{\circ}{F}_{\mathrm{SD} / \overline{\mathrm{SD}} m_{1} m_{2}} & =\stackrel{\circ}{F}_{\tau} m_{1} m_{2} \pm\left\{g \eta_{m_{1} n_{1}} \eta_{m_{2} n_{2}} \stackrel{\circ}{F}_{\sigma}^{n_{1} n_{2}}+\left(s_{\left[m_{1}\right.} \stackrel{\circ}{\sigma}_{\left.\sigma \mid m_{2}\right]}-\epsilon_{m_{1} m_{2} n_{1} n_{2} k} \bar{s}^{k} \stackrel{\circ}{F}_{\sigma}^{n_{1} n_{2}}\right)\right\} \\
\stackrel{\circ}{F}_{\mathrm{SD} / \overline{\mathrm{SD}}} & =\stackrel{\circ}{F}_{\tau} \pm\left\{g \stackrel{\circ}{F_{\sigma}}-\bar{s}^{n} \stackrel{\circ}{F}_{\sigma n}\right\}
\end{aligned}\right.
\end{aligned}
$$

where the selfdual or the anti-selfdual currents picks up the + or - among \pm sign respectively. The GL(5) covariant field strengths in flat space are given by

$$
\begin{aligned}
& \stackrel{\circ}{F}_{\tau} M=\left(\stackrel{\circ}{F}_{\tau}^{m}, \stackrel{\circ}{F}_{\tau m n}, \stackrel{\circ}{\bar{F}}_{\tau}\right) \\
& \left\{\begin{aligned}
\stackrel{\circ}{F}_{\tau}^{m} & =-\dot{X}^{m}+\partial^{m} Y \\
\stackrel{\circ}{F}_{\tau} m_{1} m_{2} & =\dot{X}_{m_{1} m_{2}}+\frac{1}{2} \epsilon_{m_{1} \cdots m_{5}} \partial^{m_{3}} Y^{m_{4} m_{5}} \\
\stackrel{\circ}{F}_{\tau} & =\dot{\bar{X}}+\partial^{m} Y_{m}
\end{aligned}\right. \\
& \stackrel{\circ}{F}_{\sigma M}=\left(\stackrel{\circ}{F}_{\sigma ; m}, \stackrel{\circ}{F}_{\sigma}^{m n}, \stackrel{\circ}{F}_{\sigma}\right) \\
& \left\{\begin{aligned}
\stackrel{\circ}{\stackrel{\circ}{F}_{\sigma ; m}} & =\partial^{n} X_{n m} \\
\stackrel{\circ}{F}_{m_{1} m_{2}} & =\partial^{\left[m_{1}\right.} X^{\left.m_{2}\right]} \\
\stackrel{\circ}{F}_{\sigma} & =0
\end{aligned}\right.
\end{aligned}
$$

The $\mathrm{SO}(5,5)$ background $G_{M N}=E_{M}{ }^{A} \hat{\eta}_{A B} E_{N}{ }^{B}$ is given by the vielbein $E_{M}{ }^{A}$ from (3.38) as

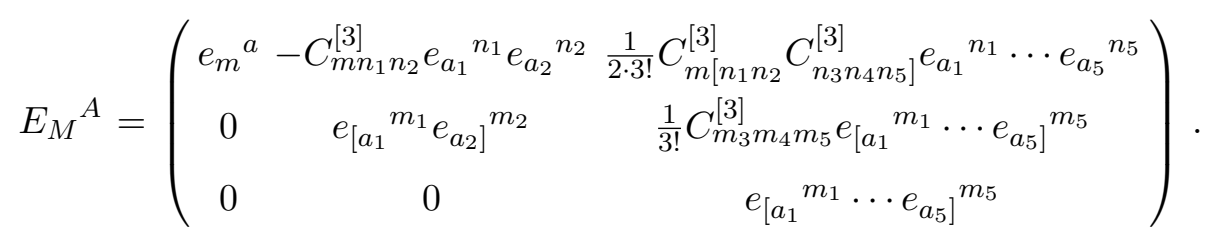

We propose a perturbative action for a M-theory 5-brane in the curved background in terms of the selfdual and the anti-selfdual currents in (4.42) as follow.

$$
\begin{aligned}
& I=\int d \tau d^{5} \sigma L
\end{aligned}
$$

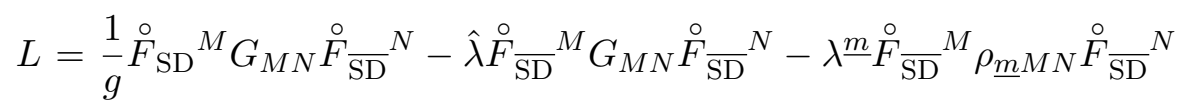

$$
\begin{aligned}
& =\frac{1}{g} F_{\mathrm{SD}}{ }^{A} \hat{\eta}_{A B} F_{\overline{\mathrm{SD}}^{B}}^{B}-\hat{\lambda} F_{\overline{\mathrm{SD}}}^{A} \hat{\eta}_{A B} F_{\overline{\mathrm{SD}}^{B}}^{B}-\lambda{ }^{\underline{a}}{\overline{\mathrm{SD}}^{A}}^{A} \underline{a}_{A B} F_{\overline{\mathrm{SD}}^{B}}^{B} .
\end{aligned}
$$

The first term is a free kinetic term for GL(5) tensor fields on a $(5+1)$-dimensional worldvolume, while the rest is constraints of the anti-selfdual currents. 


\section{Conclusions}

We have presented the F-theory 10-brane actions with the $\mathrm{SO}(5,5) \mathrm{U}$-duality symmety and the $\mathrm{SO}(6,6)$ enlarged U-duality symmetry. At first the $\mathrm{SO}(5,5)$ current algebras on the 10-dimensional worldvolume are presented in both the $\mathrm{SO}(5,5)$ spinor representation and the GL(5) tensor representation. The former reveals the gauge symmetry structure generated by the Gauss law constraint where the 10-dimensional worldvolume translation and the 16-dimensional spacetime current satisfy the bosonic $\kappa$-symmetry structure. The latter gives direct coupling to the 11-dimensional supergravity background fields. Next the action of the F-theory 10-brane is obtained by the Legendre transformation of the Hamiltonian constructed by the set of Virasoro constraints. Applying the double zweibein method to F-theory allows to give the 10-dimensional worldvolume covariant actions. Then we have also constructed the F-theory 10-brane action with the $\mathrm{SO}(6,6)$ symmetry in the Lagrangian formalism. The worldvolume is enlarged to 12 dimensional brane spacetime, while the target spacetime is enlarged to the 32 dimensional spacetime. The background vielbein represents the coset $\mathrm{SO}(6,6) / \mathrm{SO}(6 ; \mathbb{C})$ including both the spacetime background $\mathrm{SO}(5,5) / \mathrm{SO}(5 ; \mathbb{C})$ and the worldvolume vielbein.

We have also presented the action for the perturbative M-theory 5-brane in curved spacetime by sectioning the worldvolume of the F-theory 10-brane action. The spacetime is 16 dimensional manifesting the $\mathrm{SO}(5,5)$ background coupling. The action is sum of the free kinetic term and the bilinears of the selfduality constraint. So now quantization of the F10brane and the M5-brane is challenging problem. It is also interesting to note that 5-brane is the only object that appears common to all theories; type I, IIA, IIB superstrings, $\mathrm{SO}(32)$, $\mathrm{E}_{8} \times \mathrm{E}_{8}$ heterotic superstrings, M-theory, F-theory. In F-theory the 5-brane represents the SL(5) U-duality symmetry [46]. Recently it was shown that current algebras of 5-branes are preserved under the $\mathrm{S}$ and T-duality transformations with renaming the spacetime coordinates, where 5-branes include the NS5-brane, the D5-brane, KK5-branes and exotic 5-branes in 32-supersymmetric string theories [54]. 5-brane may give a clue of duality web including 16 supersymmetric string theories.

Many interesting topics are unsolved such as supersymmetric actions of F-theory and M-theory, first quantization of branes and spectrum, amplitudes, and duality web including 16-supersymmetric theories.

\section{Acknowledgments}

We would like to thank Di Wang for useful discussions. M.H. would like to thank the Simons Center for Geometry and Physics for hospitality during "the 2019 Summer Simons workshop in Mathematics and Physics" where this work has been developed. W.S. is supported by NSF grant PHY-1915093. 


\section{A Indices}

Indices are summarized as follows.

\begin{tabular}{cccc}
\hline & number of d.o.f. & curved & flat \\
\hline F-theory & & & \\
\hline spacetime & & & \\
SO(5,5) spinor & 16 & $\mu, \nu, \cdots$ & $\alpha, \beta, \cdots$ \\
SO(6,6) spinor & 32 & $\underline{\mu}, \underline{\nu}, \cdots$ & $\underline{\alpha}, \underline{\beta}, \cdots$ \\
GL(5) tensor & 16 & $M, N, \cdots$ & $A, B, \cdots$ \\
GL(6) tensor & 32 & $\underline{M}, \underline{N}, \cdots$ & $\underline{A}, \underline{B}, \cdots$ \\
\hline worldvolume & & & \\
SO(5,5) vector & 10 & $\underline{m}, \underline{n}, \cdots$ & $\underline{a}, \underline{b}, \cdots$ \\
SO $(6,6)$ vector & 12 & $\underline{\hat{m}}, \underline{\hat{n}}, \cdots$ & $\underline{\hat{a}}, \underline{\hat{b}}, \cdots$ \\
GL(5) vector & 5 & $m, n, \cdots$ & $a, b, \cdots$ \\
GL $(6)$ vector & 6 & $\hat{m}, \hat{n}, \cdots$ & $\hat{a}, \hat{b}, \cdots$ \\
\hline T-theory & & & \\
$($ only section 4.1$)$ & & & \\
\hline spacetime & & & \\
O $(D, D)$ vector & $2 D$ & $M, N, \cdots$ & $A, B, \cdots$ \\
left-handed & $D$ & $\bar{M}, \bar{N}, \cdots$ & $\bar{A}, \bar{B}, \cdots$ \\
right-handed & $D$ & $\underline{M}, \underline{N}, \cdots$ & $\underline{A}, \underline{B}, \cdots$ \\
\hline worldvolume & & & \\
SO $(1,1)$ vector & 2 & $m, n, \cdots$ & $a, b, \cdots$ \\
\hline
\end{tabular}

\section{B Brackets}

In the F-theory spacetime the Lie derivative is modified in such a way that it is the $\mathrm{SO}(5,5)$ U-duality symmetry covariant. We compute commutators in the $\mathrm{SO}(5,5)$ spinor representation which is easier than the GL(5) representation. For vector functions $V_{i}^{\mu}(X)$ with $i=1,2$ in the 16-dimensional spacetime a commutator brackets of these vectors is given by

$$
\begin{aligned}
{\left[V_{1}^{\mu} \triangleright_{\mu}(\sigma), V_{2}^{\nu} \triangleright_{\nu}\left(\sigma^{\prime}\right)\right]=} & 2 i\left(\frac{1-K}{2} \Phi_{(12)}^{\underline{m}}(\sigma)+\frac{1+K}{2} \Phi_{(12)}^{\underline{m}}\left(\sigma^{\prime}\right)\right) \partial_{\underline{m}} \delta\left(\sigma-\sigma^{\prime}\right) \\
& -i\left(V_{1}^{\nu} \partial_{\nu} V_{2}^{\mu}-V_{2}^{\nu} \partial_{\nu} V_{1}^{\mu}\right) \triangleright_{\mu} \delta\left(\sigma-\sigma^{\prime}\right) \\
& +i\left(\frac{1-K}{2} V_{1} \gamma^{\underline{m}} \partial_{\mu} V_{2}-\frac{1+K}{2} \partial_{\mu} V_{1} \gamma^{\underline{m}} V_{2}\right)\left(\gamma_{\underline{m}} \triangleright\right)^{\mu} \delta\left(\sigma-\sigma^{\prime}\right) \\
\Phi_{(12)}^{\frac{m}{1}}= & V_{1}{ }^{\mu} \gamma^{\underline{m}}{ }_{\mu \nu} V_{2}^{\nu} .
\end{aligned}
$$


The exceptional Courant bracket is given by $K=0$ as

$$
\begin{aligned}
{\left[V_{1}^{\mu} \triangleright_{\mu}(\sigma), V_{2}^{\nu} \triangleright_{\nu}\left(\sigma^{\prime}\right)\right] } & =i\left(\Phi_{(12)}^{\underline{m}}(\sigma)+\Phi \frac{m}{(12)}^{\prime}\left(\sigma^{\prime}\right)\right) \partial_{\underline{m}} \delta\left(\sigma-\sigma^{\prime}\right)-i V_{[12]}^{\mu} \triangleright_{\mu} \delta\left(\sigma-\sigma^{\prime}\right) \\
V_{[12]}^{\mu} & =V_{[1 \mid}^{\nu} \partial_{\nu} V_{\mid 2]}^{\mu}-\frac{1}{2}\left(V_{[1 \mid}^{\rho} \gamma^{\underline{m}} \rho \lambda \partial_{\nu} V_{\mid 2]}^{\lambda}\right) \gamma_{\underline{m}}^{\nu \mu} .
\end{aligned}
$$

while the exceptional Dorfman bracket is given by $K=1$ as

$$
\begin{aligned}
{\left[V_{1}^{\mu} \triangleright_{\mu}(\sigma), V_{2}^{\nu} \triangleright_{\nu}\left(\sigma^{\prime}\right)\right]=} & 2 i \Phi_{(12)}^{\frac{m}{\left(\sigma^{\prime}\right.}}\left(\partial_{\underline{m}} \delta\left(\sigma-\sigma^{\prime}\right)\right. \\
& -i\left(V_{[1 \mid}^{\nu} \partial_{\nu} V_{\mid 2]}^{\mu}+\left(\partial_{\nu} V_{1} \gamma^{\underline{m}} V_{2}\right) \gamma_{\underline{m}}^{\nu \mu}\right) \triangleright_{\mu} \delta\left(\sigma-\sigma^{\prime}\right) .
\end{aligned}
$$

\section{11-dimensional tensor representation}

The 16 component $\mathrm{SO}(5,5)$ spinor current is decomposed under the GL(5) as $16 \rightarrow 5 \oplus$ $10 \oplus 1$. We present the current algebras preserving the full tensor indices such as $\triangleright^{m_{1} \cdots m_{5}}$ with $m=1, \cdots, 5$ in order to manifest the 11-dimensional supergravity background. The $\mathrm{SO}(5,5)$ current for the M5-brane is obtained as $\triangleright_{M}=\left(\triangleright_{m}, \triangleright^{m_{1} m_{2}}, \triangleright^{m_{1} \cdots m_{5}}\right)$ [41]. The Ftheory $\mathrm{SO}(5,5)$ current algebras give in $(3.8)$ are rewritten in terms of the $\mathrm{GL}(5)$ tensors as

$$
\begin{gathered}
\left\{\begin{aligned}
{\left[\triangleright_{m}(\sigma), \triangleright^{n_{1} n_{2}}\left(\sigma^{\prime}\right)\right] } & =2 i \delta_{m}^{\left[n_{1}\right.} \partial^{\left.n_{2}\right]} \delta\left(\sigma-\sigma^{\prime}\right) \\
{\left[\triangleright_{m}(\sigma), \triangleright^{n_{1} \cdots n_{5}}\left(\sigma^{\prime}\right)\right] } & \left.=\frac{2 i}{4 !} \delta_{m}^{n_{1}} \partial^{n_{2} \cdots n_{5}}\right] \delta\left(\sigma-\sigma^{\prime}\right) \\
{\left[\triangleright^{m_{1} m_{2}}(\sigma), \triangleright^{n_{3} n_{4}}\left(\sigma^{\prime}\right)\right] } & =2 i \partial^{m_{1} m_{2} n_{3} n_{4}} \delta\left(\sigma-\sigma^{\prime}\right)
\end{aligned}\right. \\
\left\{\begin{aligned}
& {\left[\triangleright_{M}(\sigma), \tilde{\triangleright}_{N}\left(\sigma^{\prime}\right)\right]=0 } \\
& {\left[\tilde{\triangleright}_{m}(\sigma), \tilde{\triangleright}^{n_{1} n_{2}}\left(\sigma^{\prime}\right)\right] }=-2 i \delta_{m}^{\left[n_{1}\right.} \partial^{\left.n_{2}\right]} \delta\left(\sigma-\sigma^{\prime}\right) \\
& {\left[\tilde{\triangleright}_{m}(\sigma), \tilde{\triangleright}^{n_{1} \cdots n_{5}}\left(\sigma^{\prime}\right)\right] }=-\frac{2 i}{4 !} \delta_{m}^{n_{1}} \partial^{\left.n_{2} \cdots n_{5}\right]} \delta\left(\sigma-\sigma^{\prime}\right) \\
& {\left[\tilde{\triangleright}^{m_{1} m_{2}}(\sigma), \tilde{\triangleright}^{n_{3} n_{4}}\left(\sigma^{\prime}\right)\right] }=-2 i \partial^{m_{1} m_{2} n_{3} n_{4}} \delta\left(\sigma-\sigma^{\prime}\right)
\end{aligned}\right.
\end{gathered}
$$

where the $\delta(\sigma)$ stands for the 10-dimensional worldvolume function $\delta^{(10)}\left(\sigma-\sigma^{\prime}\right)$. The bosonic coordinates, $X^{M}=\left(X^{m}, X_{m_{1} m_{2}}, X_{m_{1} \cdots m_{5}}\right)$ and $P_{M}=\left(P_{m}, P^{m_{1} m_{2}}, P^{m_{1} \cdots m_{5}}\right)$, satisfy the following canonical commutators

$$
\left\{\begin{aligned}
{\left[P_{m}(\sigma), X^{n}(\sigma)\right] } & =\frac{1}{i} \delta_{m}^{n} \delta\left(\sigma-\sigma^{\prime}\right) \\
{\left[P^{m_{1} m_{2}}(\sigma), X_{n_{1} n_{2}}(\sigma)\right] } & =\frac{1}{i} \delta_{m_{1}}^{\left[n_{1}\right.} \delta_{m_{2}}^{\left.n_{2}\right]} \delta\left(\sigma-\sigma^{\prime}\right) \\
{\left[P^{m_{1} \cdots m_{5}}(\sigma), X_{n_{1} \cdots n_{5}}(\sigma)\right] } & =\frac{1}{i} \delta_{m_{1}}^{\left[n_{1}\right.} \cdots \delta_{m_{5}}^{\left.n_{5}\right]} \delta\left(\sigma-\sigma^{\prime}\right) .
\end{aligned}\right.
$$

The covariant derivatives, which are selfdual currents, are given as

$$
\left\{\begin{aligned}
\triangleright_{m} & =P_{m}+\partial^{n} X_{m n}+\frac{1}{4 !} \partial^{m_{1} \cdots m_{4}} X_{m m_{1} \cdots m_{4}} \\
\triangleright^{m_{1} m_{2}} & =P^{m_{1} m_{2}}-\partial^{\left[m_{1}\right.} X^{\left.m_{2}\right]}+\frac{1}{2} \partial^{m_{1} \cdots m_{4}} X_{m_{3} m_{4}} \\
\triangleright^{m_{1} \cdots m_{5}} & =P^{m_{1} \cdots m_{5}}+\frac{1}{4 !} \partial^{\left[m_{1} \cdots m_{4}\right.} X^{\left.m_{5}\right]}
\end{aligned}\right.
$$

and the symmetry generators, which are anti-selfdual currents, are given as

$$
\left\{\begin{aligned}
\tilde{\triangleright}_{m} & =P_{m}-\partial^{n} X_{m n}-\frac{1}{4 !} \partial^{m_{1} \cdots m_{4}} X_{m m_{1} \cdots m_{4}} \\
\tilde{\triangleright}^{m_{1} m_{2}} & =P^{m_{1} m_{2}}+\partial^{\left[m_{1}\right.} X^{\left.m_{2}\right]}-\frac{1}{2} \partial^{m_{1} \cdots m_{4}} X_{m_{3} m_{4}} \\
\tilde{\triangleright}^{m_{1} \cdots m_{5}} & =P^{m_{1} \cdots m_{5}}-\frac{1}{4 !} \partial^{\left[m_{1} \cdots m_{4}\right.} X^{\left.m_{5}\right]}
\end{aligned}\right.
$$


The Virasoro constraints are the followings

$$
\left\{\begin{aligned}
\mathcal{S}^{m}= & \frac{1}{2} \triangleright_{n} \triangleright^{n m}=0 \\
\mathcal{S}^{m_{1} \cdots m_{4}}= & \left.\frac{1}{2}\left[\triangleright_{n} \triangleright^{n m_{1} \cdots m_{4}}+\frac{1}{8} \triangleright^{\left[m_{1} m_{2}\right.} \triangleright^{m_{3} m_{4}}\right]\right]=0 \\
\mathcal{T}= & \frac{1}{4}\left[\triangleright_{m} \delta^{m n} \triangleright_{n}+\frac{1}{2} \triangleright^{m_{1} m_{2}} \delta_{m_{1} n_{1}} \delta_{m_{2} n_{2}} \triangleright^{n_{1} n_{2}}\right. \\
& \left.+\frac{1}{5 !} \triangleright^{m_{1} \cdots m_{5}} \delta_{m_{1} n_{2}} \cdots \delta_{m_{5} n_{5}} \triangleright^{n_{1} \cdots n_{5}}\right]=0
\end{aligned}\right.
$$

For the tensor calculation there are several useful relations to obtain the Virasoro algebras for the totally antisymmetric tensors $t^{m_{1} m_{2}}$ and $T^{m_{1} \cdots m_{4}}$ which are obtained by the totally anti-symmetric 6 indices as

$$
\begin{aligned}
\frac{1}{3 !} V^{\left[m_{1}\right.} \epsilon^{\left.m_{2} m_{3} m_{4}\right] n_{1} n_{2}} & =-V^{\left[n_{1}\right.} \epsilon^{\left.n_{2}\right] m_{1} m_{2} m_{3} m_{4}} \\
\frac{1}{4} t^{\left[m_{1} m_{2}\right.} T^{\left.m_{3} m_{4}\right] n_{1} n_{2}} & =-t^{n_{1} n_{2}} T^{m_{1} \cdots m_{4}} \\
\frac{1}{4} \epsilon^{m l_{1} l_{2}\left[n_{1} n_{2}\right.} T^{\left.n_{3} n_{4}\right]} & =\epsilon^{m n_{1} \cdots n_{4}} T^{l_{1} l_{2}} .
\end{aligned}
$$

\section{Double vielbein formulation}

The double vielbein formulation [10] in the simplest example is explain in this appendix. The selfduality constraint in the T-theory Hamiltonian with a flat worldsheet is the antiselfduality current is 0: the 2D-dimensional selfdual current (the covariant derivative) and the $2 \mathrm{D}$-dimensional selfduality current (the symmetry generator current) are given by

$$
\left\{\begin{array}{l}
\triangleright_{M}=P_{M}+\partial_{\sigma} X^{N} \eta_{N M} \\
\tilde{\triangleright}_{M}=P_{M}-\partial_{\sigma} X^{N} \eta_{N M} .
\end{array}\right.
$$

When the 2D-dimensional coordinate $X^{M}$ is written in terms of the D-dimensional coordinates as $(x, y)$ and the canonical conjugates as $\left(p_{x}, p_{y}\right)$ for the $\mathrm{O}(D, D)$ invariant metric $\eta_{M N}=\left(\begin{array}{ll}0 & 1 \\ 1 & 0\end{array}\right)$, the currents are written as

$$
\triangleright_{M}=\left\{\begin{array}{l}
p_{x}+\partial_{\sigma} y \\
p_{y}+\partial_{\sigma} x
\end{array}, \quad \tilde{\triangleright}_{M}=\left\{\begin{array}{l}
p_{x}-\partial_{\sigma} y \\
p_{y}-\partial_{\sigma} x
\end{array}\right.\right.
$$

The selfduality constraint is the anti-selfdual current is $0 \tilde{\triangleright}_{M}=0$ in the usual formulation. By using the selfduality constraint $p_{y}=\partial_{\sigma} x$, the selfdual current reduces into the Ddimensional momenta and the winding modes $\triangleright_{M} \rightarrow\left(p_{x}, \partial_{\sigma} x\right)$.

When the Hamiltonian is made from only the selfdual currents, the Hamiltonian form Lagrangian gives a chiral scalar Lagrangian where the term $\left(\partial_{\sigma} x\right)^{2}$ is absent.

$$
\begin{aligned}
I & =\int L, & L & =\dot{x} p-H \\
H & =\frac{g}{2} \triangleright^{2} \rightarrow L & & =\frac{1}{g}\left(\dot{X}^{2}+2 g \dot{X} \partial_{\sigma} X\right) .
\end{aligned}
$$


But adding the squared anti-selfdual current as a constraint with the Lagrange multiplier $\tilde{g}$ leads to the worldsheet covariant action as

$$
H=\frac{g}{4} \triangleright^{2}+\frac{\tilde{g}}{4} \tilde{\triangleright}^{2} \rightarrow L=\frac{1}{g+\tilde{g}}\left(\dot{X}-g \partial_{\sigma} X\right)\left(\dot{X}+\tilde{g} \partial_{\sigma} X\right) .
$$

This is rewritten in terms of the selfdual and the anti-selfdual currents as

$$
\begin{gathered}
L=\frac{1}{2 g} J_{\mathrm{SD}} J_{\overline{\mathrm{SD}}}+\frac{g-\tilde{g}}{2 g(g+\tilde{g})} J_{\overline{\mathrm{SD}}}^{2} \\
\left\{\begin{array}{l}
J_{\mathrm{SD}}{ }^{M}=\dot{X}^{M}+g \hat{\eta}^{M N} \eta_{N L} \partial_{\sigma} X^{L} \\
J_{\overline{\mathrm{SD}}}=\dot{X}^{M}-g \hat{\eta}^{M N} \eta_{N L} \partial_{\sigma} X^{L}
\end{array}\right. \\
J_{\mathrm{SD}}{ }^{M}=\left\{\begin{array}{l}
\dot{x}+g \partial_{\sigma} y \\
\dot{y}+g \partial_{\sigma} x
\end{array}, \quad J_{\overline{\mathrm{SD}}}=\left\{\begin{array}{l}
\dot{x}-g \partial_{\sigma} y \\
\dot{y}-g \partial_{\sigma} x .
\end{array}\right.\right.
\end{gathered}
$$

The first term in the Lagrangian is the free kinetic term while the second term is the selfduality constraint in a bilinear form. The bilinear form constraint reduces into the anti-selfdual current to be 0 , which relates the doubled coordinates $x$ and $y$ as the usual selfduality constraint.

Open Access. This article is distributed under the terms of the Creative Commons Attribution License (CC-BY 4.0), which permits any use, distribution and reproduction in any medium, provided the original author(s) and source are credited.

\section{References}

[1] W. Siegel, Manifest duality in low-energy superstrings, in proceedings of the International Conference on Strings 93, Berkeley, California, U.S.A., 24-29 May 1993, pp. 353-363 [hep-th/9308133] [INSPIRE].

[2] W. Siegel, Superspace duality in low-energy superstrings, Phys. Rev. D 48 (1993) 2826 [hep-th/9305073] [INSPIRE].

[3] W. Siegel, Two vierbein formalism for string inspired axionic gravity, Phys. Rev. D 47 (1993) 5453 [hep-th/9302036] [INSPIRE].

[4] M. Poláček and W. Siegel, Natural curvature for manifest T-duality, JHEP 01 (2014) 026 [arXiv:1308.6350] [INSPIRE].

[5] M. Hatsuda, K. Kamimura and W. Siegel, Ramond-Ramond gauge fields in superspace with manifest T-duality, JHEP 02 (2015) 134 [arXiv:1411.2206] [InSPIRE].

[6] M. Hatsuda, K. Kamimura and W. Siegel, Superspace with manifest T-duality from type-II superstring, JHEP 06 (2014) 039 [arXiv:1403.3887] [INSPIRE].

[7] M. Poláček and W. Siegel, T-duality off shell in 3D Type II superspace, JHEP 06 (2014) 107 [arXiv: 1403.6904] [INSPIRE].

[8] M. Hatsuda, K. Kamimura and W. Siegel, Type II chiral affine Lie algebras and string actions in doubled space, JHEP 09 (2015) 113 [arXiv:1507.03061] [INSPIRE].

[9] M. Hatsuda, K. Kamimura and W. Siegel, Manifestly T-dual formulation of AdS space, JHEP 05 (2017) 069 [arXiv:1701.06710] [InSPIRE]. 
[10] M. Hatsuda and W. Siegel, $O(D, D)$ gauge fields in the T-dual string Lagrangian, JHEP 02 (2019) 010 [arXiv: 1810.04761] [INSPIRE].

[11] M. Hatsuda and W. Siegel, T-dual Superstring Lagrangian with double Zweibeins, JHEP 03 (2020) 058 [arXiv: 1912.05092] [INSPIRE].

[12] C. Hull and B. Zwiebach, Double Field Theory, JHEP 09 (2009) 099 [arXiv:0904.4664] [INSPIRE].

[13] C. Hull and B. Zwiebach, The Gauge algebra of double field theory and Courant brackets, JHEP 09 (2009) 090 [arXiv:0908.1792] [INSPIRE].

[14] B. Zwiebach, Double Field Theory, T-duality, and Courant Brackets, in Lecture Notes in Physics 851, Springer (2012), pp. 265-291 [arXiv:1109.1782] [INSPIRE].

[15] D.S. Berman and D.C. Thompson, Duality Symmetric String and M-theory, Phys. Rept. 566 (2014) 1 [arXiv:1306.2643] [INSPIRE].

[16] G. Aldazabal, D. Marques and C. Núñez, Double Field Theory: A Pedagogical Review, Class. Quant. Grav. 30 (2013) 163001 [arXiv: 1305.1907] [InSPIRE].

[17] O. Hohm, D. Lüst and B. Zwiebach, The Spacetime of Double Field Theory: Review, Remarks, and Outlook, Fortsch. Phys. 61 (2013) 926 [arXiv:1309.2977] [InSPIRE].

[18] J.-H. Park, Green-Schwarz superstring on doubled-yet-gauged spacetime, JHEP 11 (2016) 005 [arXiv: 1609.04265] [INSPIRE].

[19] N. Hitchin, Generalized Calabi-Yau manifolds, Quart J. Math. Oxford 54 (2003) 281 [math/0209099].

[20] N. Hitchin, Brackets, forms and invariant functionals, math/0508618 [INSPIRE].

[21] M. Gualtieri, Generalized complex geometry, Ph.D. Thesis, University of Oxford, Oxford U.K. (2003) [math/0401221] [INSPIRE].

[22] C.M. Hull and P.K. Townsend, Unity of superstring dualities, Nucl. Phys. B 438 (1995) 109 [hep-th/9410167] [INSPIRE].

[23] E. Witten, String theory dynamics in various dimensions, Nucl. Phys. B 443 (1995) 85 [hep-th/9503124] [INSPIRE].

[24] C. Vafa, Evidence for F-theory, Nucl. Phys. B 469 (1996) 403 [hep-th/9602022] [inSPIRE].

[25] M.P. Blencowe and M.J. Duff, Supermembranes and the Signature of Space-time, Nucl. Phys. $B 310$ (1988) 387 [INSPIRE].

[26] C.M. Hull, String dynamics at strong coupling, Nucl. Phys. B 468 (1996) 113 [hep-th/9512181] [INSPIRE].

[27] C.M. Hull, Generalised Geometry for M-theory, JHEP 07 (2007) 079 [hep-th/0701203] [INSPIRE].

[28] P. Pires Pacheco and D. Waldram, M-theory, exceptional generalised geometry and superpotentials, JHEP 09 (2008) 123 [arXiv:0804.1362] [INSPIRE].

[29] D.S. Berman and M.J. Perry, Generalized Geometry and M-theory, JHEP 06 (2011) 074 [arXiv: 1008.1763] [INSPIRE].

[30] D.S. Berman, H. Godazgar and M.J. Perry, $\mathrm{SO}(5,5)$ duality in M-theory and generalized geometry, Phys. Lett. B $\mathbf{7 0 0}$ (2011) 65 [arXiv:1103.5733] [INSPIRE].

[31] O. Hohm and H. Samtleben, Exceptional Field Theory I: $E_{6(6)}$ covariant Form of M-theory and Type IIB, Phys. Rev. D 89 (2014) 066016 [arXiv:1312.0614] [InSPIRE]. 
[32] O. Hohm and H. Samtleben, Exceptional field theory. II. E $E_{7(7)}$, Phys. Rev. D 89 (2014) 066017 [arXiv: 1312.4542] [INSPIRE].

[33] O. Hohm and H. Samtleben, Exceptional field theory. III. E 8(8) , Phys. Rev. D 90 (2014) 066002 [arXiv: 1406 .3348] [INSPIRE].

[34] A. Coimbra, C. Strickland-Constable and D. Waldram, $E_{d(d)} \times \mathbb{R}^{+}$generalised geometry, connections and M-theory, JHEP 02 (2014) 054 [arXiv:1112.3989] [INSPIRE].

[35] D.S. Berman, M. Cederwall, A. Kleinschmidt and D.C. Thompson, The gauge structure of generalised diffeomorphisms, JHEP 01 (2013) 064 [arXiv: 1208.5884] [INSPIRE].

[36] H. Godazgar, M. Godazgar, O. Hohm, H. Nicolai and H. Samtleben, Supersymmetric $E_{7(7)}$ Exceptional Field Theory, JHEP 09 (2014) 044 [arXiv: 1406.3235] [INSPIRE].

[37] E.T. Musaev, Exceptional field theory: SL(5), JHEP 02 (2016) 012 [arXiv:1512.02163] [INSPIRE].

[38] A. Abzalov, I. Bakhmatov and E.T. Musaev, Exceptional field theory: $\mathrm{SO}(5,5)$, JHEP 06 (2015) 088 [arXiv: 1504.01523] [InSPIRE].

[39] M. Hatsuda and T. Kimura, Canonical approach to Courant brackets for D-branes, JHEP 06 (2012) 034 [arXiv:1203.5499] [InSPIRE].

[40] M. Hatsuda and K. Kamimura, SL(5) duality from canonical M2-brane, JHEP 11 (2012) 001 [arXiv: 1208.1232] [INSPIRE].

[41] M. Hatsuda and K. Kamimura, M5 algebra and SO(5,5) duality, JHEP 06 (2013) 095 [arXiv: 1305.2258] [INSPIRE].

[42] W.D. Linch III and W. Siegel, F-brane Dynamics, arXiv: 1610.01620 [INSPIRE].

[43] C.-Y. Ju and W. Siegel, Gauging Unbroken Symmetries in F-theory, Phys. Rev. D 94 (2016) 106004 [arXiv: 1607.03017] [INSPIRE].

[44] W.D. Linch III and W. Siegel, Critical Super F-theories, arXiv:1507.01669 [INSPIRE].

[45] W.D. Linch III and W. Siegel, F-theory with Worldvolume Sectioning, JHEP 04 (2021) 022 [arXiv: 1503.00940] [INSPIRE].

[46] W.D. Linch III and W. Siegel, F-theory from Fundamental Five-branes, JHEP 02 (2021) 047 [arXiv: 1502.00510] [INSPIRE].

[47] W.D. Linch III and W. Siegel, F-theory Superspace, arXiv:1501.02761 [InSPIRE].

[48] W. Siegel, F-theory with zeroth-quantized ghosts, arXiv:1601.03953 [INSPIRE].

[49] W. Siegel and D. Wang, F-theory superspace backgrounds, arXiv:1910.01710 [INSPIRE].

[50] W. Siegel and D. Wang, Enlarged exceptional symmetries of first-quantized F-theory, arXiv: 1806.02423 [INSPIRE].

[51] W. Siegel and D. Wang, $M$ Theory from F-theory, arXiv:2010.09564 [INSPIRE].

[52] P. Pasti, D.P. Sorokin and M. Tonin, Covariant action for a $D=11$ five-brane with the chiral field, Phys. Lett. B 398 (1997) 41 [hep-th/9701037] [INSPIRE].

[53] W. Siegel, Manifest Lorentz Invariance Sometimes Requires Nonlinearity, Nucl. Phys. B 238 (1984) 307 [INSPIRE].

[54] M. Hatsuda, S. Sasaki and M. Yata, Five-brane current algebras in type-II string theories, JHEP 03 (2021) 298 [arXiv:2011.13145] [INSPIRE]. 